\title{
The tetrahexahedric angular Calogero model
}

\author{
Francisco Correa ${ }^{a, b}$ and Olaf Lechtenfeld ${ }^{b}$ \\ ${ }^{a}$ Centro de Estudios Científicos (CECs), \\ Casilla 1468, Valdivia, Chile \\ ${ }^{b}$ Institut für Theoretische Physik and Riemann Center for Geometry and Physics, \\ Leibniz Universität Hannover, \\ Appelstraße 2, 30167 Hannover, Germany \\ E-mail: correa@cecs.cl, lechtenf@itp.uni-hannover.de
}

ABstract: The spherical reduction of the rational Calogero model (of type $A_{n-1}$ and after removing the center of mass) is considered as a maximally superintegrable quantum system, which describes a particle on the $(n-2)$-sphere subject to a very particular potential. We present a detailed analysis of the simplest non-separable case, $n=4$, whose potential is singular at the edges of a spherical tetrahexahedron. A complete set of independent conserved charges and of Hamiltonian intertwiners is constructed, and their algebra is elucidated. They arise from the ring of polynomials in Dunkl-deformed angular momenta, by classifying the subspaces invariant and antiinvariant under all Weyl reflections, respectively.

Keywords: Integrable Field Theories, Conformal and W Symmetry, Discrete and Finite Symmetries, Integrable Hierarchies

ARXiv EPrint: 1508.04925 


\section{Contents}

1 Introduction and summary 1

2 The angular (relative) rational Calogero model 3

3 Warmup: the hexagonal or Pöschl-Teller model 10

4 Tetrahexahedric model: the spectrum $\quad 14$

5 Tetrahexahedric model: intertwiners \& integrability $\quad 17$

$\begin{array}{llr}6 & \text { Outlook } & 21\end{array}$

A Complementary formulae $\quad 22$

\section{Introduction and summary}

The Calogero (or Calogero-Moser) model $[1-3]^{1}$ is the paradigmatical $n$-particle integrable system in one space dimension. Originally defined for the root system of $A_{1} \oplus A_{n-1}$, the Calogero model was quickly generalized for any finite Coxeter group of rank $n$ [4]. In particular the rational version is remarkable for its conformal properties and its maximal superintegrability $[8,9]$ (see also [10]). Moreover, when the coupling constant $g$ is integral, the quantum model enjoys additional and algebraically independent conserved quantities, which make it 'analytically integrable' [11, 12]. In this case, intertwining operators relate the energy spectrum with that of the free theory (at $g=0$ or $g=1$ ). This structure was worked out in the 90s by a Russian group [11, 12] using Darboux dressing and by Dutch mathematicians [13-15] employing Dunkl operators [16, 17].

Maximal superintegrability for a quantum system of phase-space dimension $2 n$ has been characterized in the literature; for a recent account see [18] where it is termed 'maximal degenerate integrability'. The Hamiltonian $H$ is part of a rank- $n$ abelian algebra of Liouville charges (commuting Hamiltonians), but this is embedded in a larger commutant of $H$, which is spanned by the Liouville charges plus $n-1$ additional integrals of motion (Wojciechowski charges). The latter do not commute with one another but form a polynomial algebra [9] together with the Liouville charges. Denoting the commutant generators by $C$ and distinguishing between Liouville charges $C^{\prime}$ and Wojciechowski charges $C^{\prime \prime}$ so that $\{C\}=\left\{C^{\prime}, C^{\prime \prime}\right\}$, one has (see, e.g. [19])

$$
\left[C^{\prime}, C^{\prime}\right]=0, \quad\left[C^{\prime \prime}, C^{\prime}\right]=\mathcal{A}\left(C^{\prime}, C^{\prime \prime}\right), \quad\left[C^{\prime \prime}, C^{\prime \prime}\right]=\mathcal{B}\left(C^{\prime}, C^{\prime \prime}\right),
$$

\footnotetext{
${ }^{1}$ For reviews, see e.g. [4-7].
} 
where $\mathcal{A}$ and $\mathcal{B}$ are polynomial in $C^{\prime}$ and linear in $C^{\prime \prime}$, and we have suppressed the counting labels. This implies that each $H$ eigenspace splits into simultaneous eigenspaces of the other $n-1$ Liouville charges. Hence, we can find an $H$ eigenbasis labelled by $n$ quantum numbers $\left(\ell_{\alpha}\right)$ with $\alpha=1, \ldots, n$. Furthermore, these simultaneous eigenspaces carry a representation of the algebra (1.1), which governs their degeneracies.

Since the rational Calogero Hamiltonian is part of an $\operatorname{SL}(2, \mathbb{R})$ conformal algebra, it is natural to reduce the rational model to an integrable system on a sphere. Restricting ourselves to the customary $A_{1} \oplus A_{n-1}$ model, one may employ the translational invariance and firstly separate the $A_{1}$ part representing the center-of-mass motion. The relative-motion Hamiltonian may be interpreted as ruling a single particle in $\mathbb{R}^{n-1}$, subject to a potential which is singular at the reflection hyperplanes and decays like $r^{-2}$ with distance $r$ from the origin. Secondly, this radial scaling suggests a further codimension-one reduction to $S^{n-2}$, which defines the 'angular (relative) rational Calogero model'. ${ }^{2}$ This system describes a particle moving on $S^{n-2}$ in a collection of $\frac{1}{2} n(n-1)$ Higgs-oscillator potentials [20, 21], each one centered at a positive root of $A_{n-1}$ (and its antipode) and blowing up at the intersection of the reflection hyperplane with the unit sphere. These singular loci tessellate the $(n-1)$-sphere into $n$ ! hyperspherical simplices in which the particle is trapped.

A number of classical features of angular rational Calogero model (center-of-massreduced or not) have been recently analyzed by an Armenian group [22-27]. Intertwining relations for the quantum model were investigated by M. Feigin already in [28], but more recently the energy spectra and eigenstates have been constructed [29], and the algebra of angular Dunkl operators has been studied [30]. These partial results leave open the following questions:

- What is a complete set of algebraically independent conserved charges?

- Can one construct a subset of Liouville charges (in mutual involution)?

- What is a complete set of algebraically independent Hamiltonian intertwiners?

- Can one find additional conserved quantities which get intertwined?

- What kind of algebra is generated by the conserved charges and intertwiners?

- Are these models maximally quantum super- or even analytically integrable?

The answers are not easily obtained for the angular Calogero models because they are linked to nontrivial mathematical problems. For instance, the commutant of $H$ is the ring of all Weyl-invariant polynomials in the angular Dunkl operators (for a definition see the following section), and it is not obvious how to construct a minimal set of algebraically independent generators. Moreover, to establish the superintegrability one should find the maximal abelian subalgebras (i.e. the Liouville charges) in this ring.

Lacking a general strategy to attack these issues, we take an explicit look at the simplest examples, $n=3$ and $n=4$. The first one, leading to a rank-one angular system, is

\footnotetext{
${ }^{2}$ It is investigated already in the appendices of [2] (see also [1, 3] for $n=3$ ).
} 
quite trivial but well known, while the second one is still tractable and serves to illustrate most of the general concepts. Therefore, in this paper, we exemplarily analyze the ranktwo quantum system which is obtained from the four-particle rational Calogero model, via translational reduction to $\mathbb{R}^{3}$ and then radial reduction to $S^{2}$. It describes a particle on the two-sphere trapped in one of 24 right isosceles spherical triangles which make up the spherical projection of a so-called tetrahexahedron.

After presenting general (and mostly previous) results on the $A_{n-1}$ angular models in section 2 , the essentially trivial $n=3$ case, known as the Pöschl-Teller model, is reworked in section 3 as a warmup example. section 4 introduces the $n=4$ model and recapitulates its energy spectrum and eigenstates [29], before our results concerning conserved charges and intertwiners are collected in section 5 . There, we establish that any conserved charge of the tetrahexahedric angular Calogero model is built from two independent operators, say $J_{4}$ and $J_{6}$ (besides the Hamiltonian), and that this system features two independent Hamiltonian intertwiners, which we denote by $M_{3}$ and $M_{6}$. We provide explicit expressions for both and work out their (non-standard) intertwining relations with the elementary conserved charges. Since one cannot produce two commuting words with $J_{4}$ and $J_{6}$, the model enjoys only two charges in involution but, given three independent conserved quantities, it is maximally superintegrable. For integer value of the coupling, an additional independent conserved charge introduces a $\mathbb{Z}_{2}$ grading (in the spirit of 'nonlinear supersymmetry' without fermionlike degrees of freedom [31,32]) and renders the model analytically integrable. Various interesting algebraic relations are collected in the appendix.

\section{The angular (relative) rational Calogero model}

We parametrize the $n$-particle quantum phase space with coordinates $x^{\mu}$ and momenta $p_{\nu}$, subject to the canonical commutation relations (setting $\hbar=1$ )

$$
\left[x^{\mu}, p_{\nu}\right]=\mathrm{i} \delta_{\nu}^{\mu} \quad \text { with } \quad \mu, \nu=1, \ldots, n .
$$

The center-of-mass coordinate and momentum,

$$
X=\frac{1}{n} \sum_{\mu} x^{\mu} \quad \text { and } \quad P=\sum_{\nu} p_{\nu} \quad \Rightarrow \quad[X, P]=\mathrm{i},
$$

decouple in the rational Calogero Hamiltonian

$$
H_{\text {cal }}=\frac{1}{2} \sum_{\nu} p_{\nu}^{2}+\sum_{\mu<\nu} \frac{g(g-1)}{\left(x^{\mu}-x^{\nu}\right)^{2}}=\frac{1}{2 n} P^{2}+H
$$

and will be ignored in our starting Hamiltonian

$$
H=\sum_{\mu<\nu}\left\{\frac{1}{2 n}\left(p_{\mu}-p_{\nu}\right)^{2}+\frac{g(g-1)}{\left(x^{\mu}-x^{\nu}\right)^{2}}\right\}
$$

which describes the relative motion of $\left(A_{n-1}\right)$ Calogero particles on the real line, or of a single quantum particle in $\mathbb{R}^{n-1}$ subject to a potential singular at the $A_{n-1}$ Weyl chamber 
walls. The Hamiltonian is invariant under the reflection $g \rightarrow 1-g$ of the real coupling $g$, but higher conserved charges and intertwiners will not share this property.

Since this Hamiltonian is scale covariant, it pays to introduce on $\mathbb{R}^{n-1}$ polar coordinates $(r, \vec{\theta})$ with a (relative) radius $r$ and $n-2$ angles $\vec{\theta}$ together with their momenta $p_{r}$ and $L_{\vec{\theta}}$,

$$
\frac{1}{n} \sum_{\mu<\nu}\left(x^{\mu}-x^{\nu}\right)^{2}=r^{2} \quad \text { and } \quad \frac{1}{n} \sum_{\mu<\nu}\left(p_{\mu}-p_{\nu}\right)^{2}=p_{r}^{2}+\frac{1}{r^{2}} L^{2}+\frac{(n-2)(n-4)}{4 r^{2}} .
$$

The (relative) angular momentum squared $L^{2}$ is best expressed by parametrizing the relative particle motion in $\mathbb{R}^{n-1}$ with coordinates $y^{i}$ where $i=1, \ldots, n-1$,

$$
r^{2}=\sum_{i=1}^{n-1}\left(y^{i}\right)^{2}, \quad p_{i} \equiv p_{y^{i}}, \quad L_{i j}=-\mathrm{i}\left(y^{i} p_{j}-y^{j} p_{i}\right), \quad L^{2}=-\sum_{i<j} L_{i j}^{2} .
$$

Jacobi coordinates are a suitable choice for $\left\{y^{i}\right\}$. Together with a dilatation generator $D$ and a special conformal generator $K$, the Hamiltonian $H$ forms an $\mathrm{SL}(2, \mathbb{R})$ commutator algebra, as is easily seen in the polar representation

$$
H=\frac{1}{2} p_{r}^{2}+\frac{(n-2)(n-4)}{8 r^{2}}+\frac{1}{r^{2}} H_{\Omega}, \quad D=\frac{1}{2}\left(r p_{r}+p_{r} r\right), \quad K=\frac{1}{2} r^{2} .
$$

Here, the angular (relative) Hamiltonian $H_{\Omega}$ is independent of the radial coordinate or momentum, and it coincides (up to a constant shift) with the Casimir operator of the conformal SL $(2, \mathbb{R})$,

$$
H_{\Omega}=\frac{1}{2} L^{2}+U(\vec{\theta})=C-\frac{1}{8}(n-1)(n-5) \quad \text { with } \quad C=K H+H K-\frac{1}{2} D^{2} .
$$

This defines our angular Calogero system (for $A_{n-1}$ ), which describes a quantum particle on the sphere $S^{n-2}$ under the influence of the potential

$$
U(\vec{\theta})=r^{2} \sum_{\mu<\nu} \frac{g(g-1)}{\left(x^{\mu}-x^{\nu}\right)^{2}}=r^{2} \sum_{\alpha \in \mathcal{R}_{+}} \frac{g(g-1)}{(\alpha \cdot y)^{2}}=\frac{g(g-1)}{2} \sum_{\alpha \in \mathcal{R}_{+}} \cos ^{-2} \theta_{\alpha},
$$

where $\mathcal{R}_{+}$is the set of positive roots for $A_{n-1}$ and $\theta_{\alpha}$ denotes the angle (geodetic arc length on $S^{n-2}$ ) between the point $\vec{\theta}$ and the intersection of the ray in direction $\alpha$ with the unit sphere. Each of the $\frac{1}{2} n(n-1)$ contributions of $\cos ^{-2}$ form is also known as a 'Higgs oscillator' $[20,21]$. This potential is a very special one as it is tied to the root system. Its singular walls are of codimension one and tesselate the sphere into $n$ ! hyperspherical simplices.

For the spectral problem, let us pass to the position representation and encode momenta via differental operators,

$$
p_{i} \mapsto-\mathrm{i} \partial_{i} \quad \Longrightarrow \quad p_{r} \mapsto-\mathrm{i}\left(\partial_{r}+\frac{n-2}{2 r}\right)
$$

so that

$$
\begin{aligned}
H & \mapsto-\frac{1}{2}\left(\partial_{r}^{2}+\frac{n-2}{r} \partial_{r}\right)+\frac{1}{r^{2}} H_{\Omega} \quad \Leftrightarrow \\
H_{\Omega} & \mapsto r^{2} H+\frac{1}{2}\left(r \partial_{r}+n-3\right) r \partial_{r} .
\end{aligned}
$$


It is customary to remove the first-order radial derivative term via a similarity transformation,

$$
\Psi(r, \vec{\theta})=r^{-\frac{n-2}{2}} u(r, \vec{\theta}) \quad \text { and } \quad H=r^{-\frac{n-2}{2}} H^{\prime} r^{\frac{n-2}{2}},
$$

so that the Hamiltonian on $\mathbb{R}^{n-1}$ acts as follows,

$$
H^{\prime} u \mapsto-\frac{1}{2}\left(\partial_{r}^{2}-\frac{(n-2)(n-4)}{4 r^{2}}\right) u+\frac{1}{r^{2}} H_{\Omega} u .
$$

As the potential is repulsive, the spectrum is positive semi-definite and continuous,

$$
H \Psi_{E, q}=E \Psi_{E, q} \quad \Longleftrightarrow \quad H^{\prime} u_{E, q}=E u_{E, q} \quad \text { with } \quad E \in \mathbb{R}_{\geq 0},
$$

where the wave function depends on further (suppressed) quantum numbers, but $q$ parametrizes the angular Hamiltonian eigenvalues [29] (see also the appendices of [2]),

$$
H_{\Omega} v_{q}=\varepsilon_{q} v_{q} \quad \text { with } \quad \varepsilon_{q}=\frac{1}{2} q(q+n-3) \quad \text { and } \quad q \in \mathbb{R}_{\geq 0} .
$$

For vanishing potential ( $g=0$ or 1 ), $H_{\Omega}=\frac{1}{2} L^{2}$, and $q=\ell \in \mathbb{N}_{0}$ is the familiar total angular momentum for a free particle on $S^{n-2}$, with a degeneracy of $\left(\begin{array}{c}\ell+n-2 \\ n-2\end{array}\right)-\left(\begin{array}{c}\ell+n-4 \\ n-2\end{array}\right)$. The interaction greatly reduces the degeneracy and shifts this quantum number [29],

$$
q=\frac{1}{2} n(n-1) g+\ell \quad \text { and } \quad \ell=3 \ell_{3}+4 \ell_{4}+\ldots+n \ell_{n} \quad \text { with } \quad \ell_{\mu} \in \mathbb{N}_{0},
$$

where the decomposition $\ell \rightarrow\left(\ell_{3}, \ell_{4}, \ldots, \ell_{n}\right)$ into additional quantum numbers yields the correct degeneracy,

$$
\operatorname{deg}_{n}\left(\varepsilon_{q}\right)=p_{n}(\ell)-p_{n}(\ell-1)-p_{n}(\ell-2)+p_{n}(\ell-3),
$$

with $p_{n}(\ell)$ denoting the number of partitions of $\ell$ into integers not larger than $n$ or, equivalently, the number of partitions of $\ell$ into at most $n$ integers. Its generating function reads

$$
p_{n}(t):=\sum_{\ell=0}^{\infty} p_{n}(\ell) t^{\ell}=\prod_{m=1}^{n}\left(1-t^{m}\right)^{-1},
$$

and the large- $\ell$ asymptotics is $p_{n}(\ell)=\frac{\ell^{n-1}}{n !(n-1) !}+O\left(\ell^{n-2}\right)$, $\operatorname{implying} \operatorname{deg}_{n}\left(\varepsilon_{q}\right) \sim \ell^{n-2}$ for $\ell \rightarrow \infty$. Note that the degeneracy does not depend on $g$ but only on $\ell$ (for any fixed $n$ ). Up to $n=5$, fairly explicit formulae may be found [33]:

$$
\begin{aligned}
& \operatorname{deg}_{3}(\ell)=\left\{\begin{array}{ll}
0 & \text { for } \quad \ell=1,2 \bmod 3 \\
1 & \text { for } \quad \ell=0 \bmod 3
\end{array},\right. \\
& \operatorname{deg}_{4}(\ell)=\left\lfloor\frac{\ell}{12}\right\rfloor+\left\{\begin{array}{ll}
0 & \text { for } \quad \ell=1,2,5 \bmod 12 \\
1 & \text { for } \quad \ell=\text { else } \bmod 12
\end{array},\right. \\
& \operatorname{deg}_{5}(\ell)=\left\lfloor\frac{6 \ell^{2}+72 \ell-89}{720}\right\rfloor+\left\{\begin{array}{lll}
0 & \text { for } & \ell=2,22,26,46 \bmod 60 \\
2 & \text { for } & \ell=0,48 \bmod 60 \\
1 & \text { for } & \ell=\text { else } \bmod 60
\end{array} .\right.
\end{aligned}
$$


The wave functions may also be given explicitly. From

$$
\left(\partial_{r}^{2}-\left(q+\frac{n}{2}-1\right)\left(q+\frac{n}{2}-2\right) r^{-2}+2 E\right) u_{E, q}=0
$$

we read off that

$$
u_{E, q}(r, \vec{\theta})=\sqrt{r} J_{q+(n-3) / 2}(\sqrt{2 E} r) v_{q}(\vec{\theta}),
$$

revealing a radial wave function of Bessel type. The angular wave function $v_{q}(\vec{\theta})$ depends on all additional quantum numbers $\left(\ell_{3}, \ell_{4}, \ldots, \ell_{n}\right)$ and is a bit harder to find [29]. It can be constructed in the following way,

$$
v_{q}(\vec{\theta}) \equiv v_{\ell}^{(g)}(\vec{\theta}) \sim r^{n-3+q} \prod_{\mu=3}^{n}\left(\sum_{\nu=1}^{n}\left(\mathcal{D}_{\nu}\right)^{\mu}\right)^{\ell_{\mu}} \Delta^{g} r^{3-n-n(n-1) g},
$$

where

$$
\Delta=\prod_{\mu<\nu}\left(x^{\mu}-x^{\nu}\right)=\prod_{\alpha \in \mathcal{R}_{+}} \alpha \cdot y
$$

is the Vandermonde of the original $\mathbb{R}^{n}$ coordinates and $\mathcal{D}_{\nu}$ denotes the so-called Dunkl operator $[16,17]$,

$$
\mathcal{D}_{\nu}=\partial_{\nu}-g \sum_{\rho(\neq \nu)} \frac{1}{x^{\nu}-x^{\rho}} s_{\nu \rho}, \quad\left[\mathcal{D}_{\mu}, \mathcal{D}_{\nu}\right]=0,
$$

with $s_{\nu \rho}$ permuting the position and momenta of the $\nu$ th with those of the $\rho$ th particle. The Dunkl operators play a central role in asserting the integrability of the Calogero model, because deforming $\partial_{\nu} \rightarrow \mathcal{D}_{\nu}$ essentially creates the interacting system from the free one. Their Newton sums yield the Liouville charges of the Calogero system, and their mutual commutativity guarantees that of the charges.

It is convenient to switch again to the relative coordinates $\left\{y^{i}\right\}$, in which the Dunkldeformed momenta and angular momenta take the form (up to a factor of $-\mathrm{i}$ )

$$
\mathcal{D}_{i}=\partial_{i}-g \sum_{\alpha \in \mathcal{R}_{+}} \frac{\alpha_{i}}{\alpha \cdot y} s_{\alpha} \quad \text { and } \quad \mathcal{L}_{i j}=-\left(y^{i} \mathcal{D}_{j}-y^{j} \mathcal{D}_{i}\right),
$$

respectively, where $s_{\alpha}$ is the Weyl reflection on the hyperplane orthogonal to the root $\alpha$. Polynomials in the Dunkl operators are not just differential operators but also act by Weyl reflections. Their restriction to functions totally symmetric under the Weyl group is called 'residue' (denoted by 'res') and produces a purely differential operator. In this way, the Hamiltonian can be obtained from the homogeneous symmetric polynomial of degree two,

$$
\mathcal{H}=-\frac{1}{2 n} \sum_{\mu<\nu}\left(\mathcal{D}_{\mu}-\mathcal{D}_{\nu}\right)^{2}=-\frac{1}{2} \sum_{i} \mathcal{D}_{i}^{2} \quad \Longrightarrow \quad H=\operatorname{res}(\mathcal{H})
$$

Analogously, the symmetric quadratic polynomial in the angular Dunkl operators $\mathcal{L}_{i j}$ shifted by a certain pure-reflection term,

$$
\mathcal{H}_{\Omega}=-\frac{1}{2} \sum_{i<j} \mathcal{L}_{i j}^{2}+\frac{1}{2} S(S+n-3) \quad \text { with } \quad S=g \sum_{\alpha} s_{\alpha},
$$


provides the angular Hamiltonian by taking the residue $[30],{ }^{3}$

$$
H_{\Omega}=\operatorname{res}\left(\mathcal{H}_{\Omega}\right)=\frac{1}{2} \operatorname{res}\left(\mathcal{L}^{2}\right)+\frac{1}{2} \frac{n(n-1)}{2} g\left(\frac{n(n-1)}{2} g+n-3\right)=\frac{1}{2} \operatorname{res}\left(\mathcal{L}^{2}\right)+\varepsilon_{q}(\ell=0) .
$$

We remark that the residue of the pure-reflection term simply produces the ground-state energy $\varepsilon_{q}(\ell=0)$. Clearly, $H$ and $H_{\Omega}$ may be considered as 'Dunkl deformations' of $\left(-\frac{1}{2}\right.$ times) the Laplacian on $\mathbb{R}^{n-1}$ and $S^{n-2}$, respectively.

To express the angular wave function $v_{q}(\vec{\theta})$ in terms of the relative coordinates, we must rewrite $\Delta$ in terms of $\left\{y^{i}\right\}$ and the Newton sums of the $\mathcal{D}_{\nu}$ in (2.22) in terms of the $\mathcal{D}_{i}$, which depends on our choice for $\left\{y^{i}\right\}$. Phrased differently, we need to identify the totally Weyl-symmetric homogeneous polynomials $\sigma_{\mu}\left(\left\{\mathcal{D}_{i}\right\}\right)$ of orders $\mu=3,4, \ldots, n$, to go inside the large brackets of (2.22),

$$
v_{q}(\vec{\theta}) \equiv v_{\ell}^{(g)}(\vec{\theta}) \sim r^{n-3+q}\left(\prod_{\mu=3}^{n} \sigma_{\mu}\left(\left\{\mathcal{D}_{i}\right\}\right)^{\ell_{\mu}}\right) \Delta^{g} r^{3-n-n(n-1) g} .
$$

From the form of (2.29) one can infer that $v_{q}(\vec{\theta})$ is a ratio of a degree- $q$ homogeneous polynomial $h_{q}(y)$ in $\left\{y^{i}\right\}$ to the $q$ th power of the radial coordinate $r=\left[\sum_{i}\left(y^{i}\right)^{2}\right]^{-1 / 2}$. From (2.11), the $r$ independence of $v_{q}(\vec{\theta})$ and (2.15) it follows that the polynomial $h_{q}(y)$ is annihilated by $H$,

$$
h_{\ell}^{(g)}:=r^{q} v_{\ell}^{(g)}(\vec{\theta}) \quad \Longrightarrow \quad H h_{\ell}^{(g)}=0
$$

and hence may be viewed as a Dunkl-deformed harmonic polynomial on $R^{n-1}$. Another property visible from $(2.29)$ is that $h_{q}(y)$ contains a factor of $\Delta^{g}$, which may be split off to define

$$
\widetilde{h}_{\ell}^{(g)}:=\Delta^{-g} h_{\ell}^{(g)} \quad \Longrightarrow \quad \widetilde{H} \widetilde{h}_{\ell}^{(g)}=0,
$$

which then is in the kernel of the Hamiltonian 'in the potential-free frame',

$$
\widetilde{H}=\Delta^{-g} H \Delta^{g}=-\sum_{\mu<\nu}\left\{\frac{1}{2 n}\left(\partial_{\mu}-\partial_{\nu}\right)^{2}+\frac{g}{x^{\mu}-x^{\nu}}\left(\partial_{\mu}-\partial_{\nu}\right)\right\}
$$

The potential-free Dunkl-deformed harmonic polynomial $\widetilde{h}_{\ell}^{(g)}$ is homogeneous of degree $\ell$ only, for any value of $g$. In particular, for the ground state one has

$$
\widetilde{h}_{0}^{(g)}=1 \quad \Longrightarrow \quad h_{0}^{(g)}=\Delta^{g},
$$

and hence the full ground-state wave function is totally symmetric (antisymmetric) under particle permutations or Weyl reflections for even (odd) integer values of $g$. Since all other ingredients besides $\Delta^{g}$ in (2.22) are completely symmetric, this symmetry property of the integer- $g$ ground state extends to all excited states above it. Furthermore, the reflection symmetry $g+1 \leftrightarrow-g$ is broken since one tower of states is Weyl symmetric while the other one is antisymmetric.

\footnotetext{
${ }^{3}$ We note that $\mathcal{L}^{2}=-\sum_{i<j} \mathcal{L}_{i j}^{2}$ is a non-negative operator.
} 
It is well known [13-15] that there exists an intertwining operator $M=M^{(g)}$ which establishes an isospectrality of $H^{(g)}$ and $H^{(g+1)}$. This differential operators of order $\frac{1}{2} n(n-1)$ has the simple form

$$
M=\operatorname{res}(\mathcal{M}) \quad \text { with } \quad \mathcal{M}=\prod_{\mu<\nu}\left(\mathcal{D}_{\mu}-\mathcal{D}_{\nu}\right)=\prod_{\alpha \in \mathcal{R}_{+}} \alpha \cdot \mathcal{D},
$$

and using the Weyl antisymmetry of $\mathcal{M}$ it is straightforward to verify that (see e.g. [28])

$$
[\mathcal{M}, \mathcal{H}]=0 \quad \Longrightarrow \quad M^{(g)} H^{(g)}=H^{(g+1)} M^{(g)} \quad \text { and } \quad M^{(g)} \Psi_{E, \ell}^{(g)} \sim \Psi_{E, \ell}^{(g+1)}
$$

so that

$$
M^{(g)}: \quad(g, \ell, q) \mapsto\left(g+1, \ell, q+\frac{1}{2} n(n-1)\right)
$$

simultaneously acts on the radial wave function $\sqrt{r} J_{q+(n-3) / 2}$ and on the angular wave function $v_{\ell}^{(g)}$. Up to $n=4$ the expressions for $M^{(g)}$ were worked out explicitly in [19].

However, the angular system $H_{\Omega}$ comes with its own intertwiners $M_{s}=M_{s}^{(g)}$ of some order $s \in \mathbb{N}$, which then provides additional intertwiners for $H$. This is suggested by the form (2.16) of the quantum number $q$ appearing in (2.15), which reveals a partial ${ }^{4}$ isospectrality for $H_{\Omega}$,

$$
M_{s}^{(g)}: \quad(g, \ell, q) \mapsto\left(g+1, \ell-\frac{1}{2} n(n-1), q\right) .
$$

Indeed, as shown in [28], one can more or less copy the strategy from $\mathbb{R}^{n-1}$ to $S^{n-2}$ and obtain the lowest-order angular intertwiner, with $s=\frac{1}{2}(n-1)(n-2)$,

$$
\begin{aligned}
M_{\Omega} & \equiv M_{\frac{1}{2}(n-1)(n-2)}=\operatorname{res}\left(\mathcal{M}_{\Omega}\right) \quad \text { with } \\
\mathcal{M}_{\Omega} & =\sum_{\pi \in \mathcal{S}_{n}} \operatorname{sgn}(\pi) \pi\left(\prod_{2 \leq \mu<\nu \leq n}\left\{\left(x^{1}-x^{\mu}\right)\left(\mathcal{D}_{1}-\mathcal{D}_{\nu}\right)-\left(x^{1}-x^{\nu}\right)\left(\mathcal{D}_{1}-\mathcal{D}_{\mu}\right)\right\}\right) \\
& =\sum_{\pi \in \mathcal{S}_{n}} \operatorname{sgn}(\pi) \pi\left(\prod_{2 \leq \mu<\nu \leq n}\left\{\mathcal{L}_{\mu 1}+\mathcal{L}_{1 \nu}-\mathcal{L}_{\mu \nu}\right\}\right)
\end{aligned}
$$

where the choice of $x^{1}$ as a reference is arbitrary and irrelevant. Because $\mathcal{H}$ is in the center of the algebra generated by $\left\{\mathcal{L}_{i j}\right\}$ and $\mathcal{M}_{\Omega}$ is again antisymmetric under Weyl reflections, one argues, analogously to $(2.35)$ and with the help of $H^{(-g)}=H^{(g+1)}$, that [28]

$$
\left[\mathcal{L}_{i j}, \mathcal{H}\right]=0 \Longrightarrow M_{\Omega}^{(g)} H^{(g)}=H^{(g+1)} M_{\Omega}^{(g)} \quad \text { and } \quad M_{\Omega}^{(g)} \Psi_{E, \ell}^{(g)} \sim \Psi_{E, \ell-n(n-1) / 2}^{(g+1)} .
$$

These properties hold not only for $M_{\Omega}$, but actually any Weyl-antiinvariant function of $\left\{\mathcal{L}_{i j}\right\}$ provides an angular intertwiner $M_{s}^{(g)}$ which obeys (2.39). By the same token, any Weyl-invariant function of $\left\{\mathcal{L}_{i j}\right\}$ will yield, via its residue, a conserved angular quantity $C_{t}$ of some order $t$. We remark that the angular Dunkl operators do not commute with each other but form a subalgebra of a rational Cherednik algebra [30]. It is a deformation of the $s o(n-1)$ Lie algebra generated by $\left\{L_{i j}\right\}$. The general problem of identifying all Liouville charges for the angular system reduces to constructing a maximal abelian subalgebra

\footnotetext{
${ }^{4}$ Meaning that the intertwiners have a nontrivial kernel.
} 
in the algebra of Weyl-symmetric polynomials in $\left\{\mathcal{L}_{i j}\right\}$, which is not an easy task. Likewise, identifying the minimal independent set of Weyl-antiinvariants and hence intertwiners is nontrivial.

Now, since any such $M_{s}$ and $C_{t}$ is scale invariant, i.e. $r$ independent, it does not touch the radial wave function, and hence it is also true that

$$
\begin{aligned}
& {\left[\mathcal{L}_{i j}, \mathcal{H}_{\Omega}\right]=0 \quad \Longrightarrow \quad M_{s}^{(g)} H_{\Omega}^{(g)}=H_{\Omega}^{(g+1)} M_{s}^{(g)} \quad \text { and } \quad M_{s}^{(g)} v_{\ell}^{(g)} \sim v_{\ell-n(n-1) / 2}^{(g+1)}} \\
& \text { as well as } \quad C_{t}^{(g)} H_{\Omega}^{(g)}=H_{\Omega}^{(g)} C_{t}^{(g)} \text {. }
\end{aligned}
$$

To summarize, we have the connection

$$
\begin{aligned}
\mathcal{C}_{t}\left(\mathcal{L}_{i j}\right) \quad \text { Weyl-invariant } & \Longrightarrow \quad C_{t}=\operatorname{res}\left(\mathcal{C}_{t}\right) \quad \text { commutes with } H_{\Omega} \\
\mathcal{M}_{s}\left(\mathcal{L}_{i j}\right) \quad \text { Weyl-antiinvariant } & \Longrightarrow \quad M_{s}=\operatorname{res}\left(\mathcal{M}_{s}\right) \quad \text { intertwines with } H_{\Omega} .
\end{aligned}
$$

In general, the angular conserved charges $C_{t}$ will not be in involution with one another, but some combinations may be. The angular intertwiners $M_{s}$ are differential operators of order $s$ and have, in contrast to $M$, a sizeable kernel, with

$$
\operatorname{dim} \operatorname{ker}_{n}(\ell)=\operatorname{deg}_{n}(\ell)-\operatorname{deg}_{n}\left(\ell-\frac{1}{2} n(n-1)\right) .
$$

For small values of $n$, we can be explicit:

$$
\begin{aligned}
& \operatorname{dim} \operatorname{ker}_{3}(\ell)=\left\{\begin{array}{ll}
1 & \text { for } \quad \ell=0 \\
0 & \text { for } \quad \ell>0
\end{array},\right. \\
& \operatorname{dim} \operatorname{ker}_{4}(\ell)=\left\{\begin{array}{ll}
1 & \text { for } \quad \ell=0,3 \bmod 4 \\
0 & \text { for } \quad \ell=1,2 \bmod 4
\end{array},\right. \\
& \operatorname{dim} \operatorname{ker}_{5}(\ell)=\left\lfloor\frac{\ell}{6}\right\rfloor+\left\{\begin{array}{ll}
1 & \text { for } \quad \ell=0,3,4,5,8,9,11 \bmod 12 \\
0 & \text { for } \quad \ell=1,2,6,7,10 \bmod 12
\end{array} .\right.
\end{aligned}
$$

With the help of the adjoint intertwiners, which reverse the direction of $M$ resp. $M_{s}$,

$$
M_{s}^{(g)^{\dagger}}=M_{s}^{(-g)} \quad \Longrightarrow \quad M_{s}^{(-g)} H_{\Omega}^{(g+1)}=H_{\Omega}^{(g)} M_{s}^{(-g)} \quad \text { and } \quad M_{s}^{(-g)} v_{\ell}^{(g+1)} \sim v_{\ell+n(n-1) / 2}^{(g)},
$$

it is trivial to construct special conserved charges,

$$
R_{2 s}^{(g)}:=M_{s}^{(-g)} M_{s}^{(g)} \quad \text { and } \quad R_{2 s}^{(1-g)}=M_{s}^{(g-1)} M_{s}^{(1-g)},
$$

which provide particular cases of some $C_{t}$ for $t=2 \mathrm{~s}$. In the full Calogero model, $R=M^{\dagger} M$ turned out to be a specific polynomial in the Liouville charges, which was evaluated by infinitely separating the particles thus making contact with the free case. This move is not possible for $R_{2 s}$ due to the compactness of $S^{n-2}$. Neither is it true that $R_{2 s}^{(-g)}=R_{2 s}^{(g+1)}$, and so these charges intertwine as follows,

$$
M_{s}^{(g)} R_{2 s}^{(g)}=R_{2 s}^{(-g)} M_{s}^{(g)}=\left(R_{2 s}^{(g+1)}-(1+2 g) \rho_{2 s}^{(g+1)}\right) M_{s}^{(g)},
$$


and similarly in the opposite direction. Here, $\rho_{2 s}$ is a particular expression in other conserved charges.

Quite generally, we cannot expect conserved charges other than the angular Hamiltonian to be invariant under $g \rightarrow 1-g$, and in fact one finds that

$$
C_{t}^{(-g)} \neq C_{t}^{(g+1)} \quad \text { and } \quad M_{s}^{(g)} C_{t}^{(g)} \neq C_{t}^{(g+1)} M_{s}^{(g)} .
$$

Therefore, higher conserved quantities $C_{t}$ intertwine in a more complicated fashion,

$$
M_{s}^{(g)} C_{t}^{(g)}=\sum_{s^{\prime}, t^{\prime}} \Gamma_{s t}^{s^{\prime} t^{\prime}}(g) C_{t^{\prime}}^{(g+1)} M_{s^{\prime}}^{(g)}
$$

where $\Gamma_{s t}^{s^{\prime} t^{\prime}}(g)$ are certain polynomials in $g$ of order $s+t-s^{\prime}-t^{\prime}-1$ at the most. The sum runs over all linearly independent conserved quantities including

$$
C_{2}^{(g)}=-\operatorname{res}\left(\mathcal{L}^{2}\right) \quad \text { and } \quad C_{0}^{(g)}:=1 .
$$

When the coupling $g$ is an integer, repreated intertwining relates all quantities with their analogs in the free theory $(g=0$ or 1$)$, which allows one to generate analytic expressions for all wave functions. Moreover, in this case additional conserved charges make the model 'analytically integrable' and produce a 'supercomplete' graded ring of commuting differential operators $[11,12,19]$.

\section{Warmup: the hexagonal or Pöschl-Teller model}

Let us work out and test the general assertions of the previous section on the simplest nontrivial case, which occurs for $n=3$. The three-particle (or $A_{2}$ ) rational Calogero model is long known to be completely separable, the ensuing angular (relative) model being the famed Pöschl-Teller model of a particle on a circle with a $\cos ^{-2}(3 \phi)$ potential.

We start by introducing Jacobi coordinates $\left(y^{1}, y^{2}\right)$ for the relative motion (and the center of mass $X$ ),

$$
\begin{aligned}
x^{1} & =X+\frac{1}{\sqrt{2}} y^{1}+\frac{1}{\sqrt{6}} y^{2}, & \partial_{x^{1}} & =\frac{1}{3} \partial_{X}+\frac{1}{\sqrt{2}} \partial_{y^{1}}+\frac{1}{\sqrt{6}} \partial_{y^{2}}, \\
x^{2} & =X-\frac{1}{\sqrt{2}} y^{1}+\frac{1}{\sqrt{6}} y^{2}, & \partial_{x^{2}} & =\frac{1}{3} \partial_{X}-\frac{1}{\sqrt{2}} \partial_{y^{1}}+\frac{1}{\sqrt{6}} \partial_{y^{2}}, \\
x^{3} & =X-\frac{2}{\sqrt{6}} y^{2}, & \partial_{x^{3}} & =\frac{1}{3} \partial_{X}-\frac{2}{\sqrt{6}} \partial_{y^{2}},
\end{aligned}
$$

and then polar $(r, \phi)$ and complex $(w, \bar{w})$ coordinates on $\mathbb{R}^{2}$,

$$
y^{1}=r \cos \phi \quad \text { and } \quad y^{2}=r \sin \phi \quad \Longrightarrow \quad w:=y^{1}+\mathrm{i} y^{2}=r \mathrm{e}^{\mathrm{i} \phi} .
$$

After the standard similarity transformation, the (reduced) Hamiltonian

$$
\begin{aligned}
H & =-\frac{1}{2}\left(\partial_{1}^{2}+\partial_{2}^{2}\right)+g(g-1)\left(\frac{2}{\left(2 y^{1}\right)^{2}}+\frac{2}{\left(y^{1}-\sqrt{3} y^{2}\right)^{2}}+\frac{2}{\left(y^{1}+\sqrt{3} y^{2}\right)^{2}}\right) \\
& =-\frac{1}{2}\left(\partial_{1}^{2}+\partial_{2}^{2}\right)+\frac{9 g(g-1)\left(\left(y^{1}\right)^{2}+\left(y^{2}\right)^{2}\right)}{2\left(y^{1}\right)^{2}\left(y^{1}-\sqrt{3} y^{2}\right)^{2}\left(y^{1}+\sqrt{3} y^{2}\right)^{2}}
\end{aligned}
$$


and its angular cousin take the following form as differential operators on $\mathbb{R}^{2}$ and $S^{1}$, respectively,

$$
H^{\prime} u \mapsto-\frac{1}{2}\left(\partial_{r}^{2}+\frac{1}{4 r^{2}}\right) u+\frac{1}{r^{2}} H_{\Omega} u \quad \text { with } \quad H_{\Omega}=-\frac{1}{2} \partial_{\phi}^{2}+U,
$$

where the angular potential receives contributions from the three positive roots of $A_{2}$,

$$
U(\phi)=\frac{g(g-1)}{2}\left(\cos ^{-2}(\phi)+\cos ^{-2}\left(\phi+\frac{2 \pi}{3}\right)+\cos ^{-2}\left(\phi-\frac{2 \pi}{3}\right)\right)=\frac{9}{2} g(g-1) \cos ^{-2}(3 \phi) .
$$

The poles separate the circle into six disjoint arcs, pertaining to the hexagonal symmetry of the $A_{2}$ root system. We display the angular Hamiltonian in complex coordinates as well,

$$
H_{\Omega}=\frac{1}{2}\left(w \partial_{w}-\bar{w} \partial_{\bar{w}}\right)^{2}+g(g-1) \frac{18(w \bar{w})^{3}}{\left(w^{3}+\bar{w}^{3}\right)^{2}} .
$$

Specializing (2.15)-(2.17) to $n=3$, one finds that the angular momentum $\ell=3 \ell_{3}$ is a multiple of three and

$$
\varepsilon_{q}=\frac{1}{2} q^{2} \quad \text { with } \quad q=3 g+\ell=3\left(g+\ell_{3}\right) \quad \text { and } \quad \operatorname{deg}\left(\varepsilon_{q}\right)=1 .
$$

The $\sqrt{r}$ factor in (2.21) gets cancelled in the similarity transformation (2.12), giving

$$
\Psi_{E, q}(r, \phi)=J_{q}(\sqrt{2 E} r) v_{q}(\phi)
$$

and the angular wave function reads

$$
v_{q}(\phi) \equiv v_{\ell}^{(g)}(\phi) \sim r^{q}\left(\sum_{\nu=1}^{3} \mathcal{D}_{\nu}^{3}\right)^{\ell_{3}} \Delta^{g} r^{-6 g} \sim r^{q}\left(\mathcal{D}_{w}^{3}-\mathcal{D}_{\bar{w}}^{3}\right)^{\ell_{3}} \Delta^{g} r^{-6 g}
$$

with

$$
\Delta \sim\left(x^{1}-x^{2}\right)\left(x^{2}-x^{3}\right)\left(x^{3}-x^{1}\right) \sim r^{3} \cos (3 \phi) \sim w^{3}+\bar{w}^{3},
$$

where we have rewritten in complex coordinates. ${ }^{5}$ Owing to the $S_{3}$ Weyl group of $A_{2}$, we take profit from the complex cubic roots of unity to cast the holomorphic Dunkl operator $\mathcal{D}_{w}=\frac{1}{2}\left(\mathcal{D}_{1}-\mathrm{i} \mathcal{D}_{2}\right)$ into the simple form

$$
\mathcal{D}_{w}=\partial_{w}-g\left\{\frac{1}{w+\bar{w}} s_{0}+\frac{\rho}{\rho w+\bar{\rho} \bar{w}} s_{+}+\frac{\bar{\rho}}{\bar{\rho} w+\rho \bar{w}} s_{-}\right\} \quad \text { with } \quad \rho=\mathrm{e}^{2 \pi \mathrm{i} / 3},
$$

where the three basic Weyl reflections act as follows in the complex plane,

$$
s_{0}: w \mapsto-\bar{w}, \quad s_{+}: w \mapsto-\rho \bar{w}, \quad s_{-}: w \mapsto-\bar{\rho} \bar{w}
$$

or on the polar angle,

$$
s_{0}: \phi \mapsto \pi-\phi, \quad s_{+}: \phi \mapsto-\frac{\pi}{3}-\phi, \quad s_{-}: \phi \mapsto \frac{\pi}{3}-\phi .
$$

\footnotetext{
${ }^{5}$ We refrain from displaying $v_{q}(\phi)$ in Jacobi or polar coordinates as the expressions are not enlightening.
} 
According to (2.34), the full intertwiner is a third-order operator, ${ }^{6}$

$$
\mathcal{M} \sim\left(\mathcal{D}_{1}-\mathcal{D}_{2}\right)\left(\mathcal{D}_{2}-\mathcal{D}_{3}\right)\left(\mathcal{D}_{3}-\mathcal{D}_{1}\right) \sim \mathcal{D}_{w}^{3}+\mathcal{D}_{\bar{w}}^{3},
$$

whose evaluation eventually produces [19]

$$
\begin{aligned}
M \sim & \partial_{12} \partial_{23} \partial_{31}-\frac{2 g}{x^{12}} \partial_{23} \partial_{31}-\frac{2 g}{x^{23}} \partial_{31} \partial_{12}-\frac{2 g}{x^{31}} \partial_{12} \partial_{23} \\
& +\frac{4 g^{2}}{x^{2} x^{23}} \partial_{31}+\frac{4 g^{2}}{x^{23} x^{31}} \partial_{12}+\frac{4 g^{2}}{x^{31} x^{12}} \partial_{23}-\frac{g(g-1)}{\left(x^{12}\right)^{2}} \partial_{12}-\frac{g(g-1)}{\left(x^{23}\right)^{2}} \partial_{23}-\frac{g(g-1)}{\left(x^{31}\right)^{2}} \partial_{31} \\
& -\frac{6 g^{2}(g+1)}{x^{12} x^{23} x^{31}}+2 g(g-1)(g+2)\left(\frac{1}{\left(x^{12}\right)^{3}}+\frac{1}{\left(x^{23}\right)^{3}}+\frac{1}{\left(x^{31}\right)^{3}}\right),
\end{aligned}
$$

with the abbreviated notation $x^{\mu \nu} \equiv x^{\mu}-x^{\nu}$ and $\partial_{\mu \nu} \equiv \partial_{x^{\mu}}-\partial_{x^{\nu}}$. In complex form, it reads

$$
\begin{aligned}
M \sim & \partial_{w}^{3}+\partial_{\bar{w}}^{3}-\frac{6 g}{w^{3}+\bar{w}^{3}}\left(w^{2} \partial_{w}^{2}+w \bar{w} \partial_{w} \partial_{\bar{w}}+\bar{w}^{2} \partial_{\bar{w}}^{2}\right)+\frac{6 g(3 g-1)}{w^{3}+\bar{w}^{3}}\left(w \partial_{w}+\bar{w} \partial_{\bar{w}}\right) \\
& -\frac{9 g(g-1)}{\left(w^{3}+\bar{w}^{3}\right)^{2}}\left(w^{4} \partial_{w}+\bar{w}^{4} \partial_{\bar{w}}\right)+\frac{3 g(9 g+14)}{w^{3}+\bar{w}^{3}}-\frac{27 g(g+2)}{\left(w^{3}+\bar{w}^{3}\right)^{3}}\left(w^{6}+\bar{w}^{6}+2 g w^{3} \bar{w}^{3}\right),
\end{aligned}
$$

and in the polar representation the intertwiner becomes

$$
\begin{aligned}
M & \sim \cos 3 \phi\left\{\partial_{r}^{3}-\frac{3(3 g+1)}{r} \partial_{r}^{2}+\frac{3(9 g+1)}{r^{2}} \partial_{r}-\frac{24 g}{r^{3}}+\frac{6}{r^{2}} H_{\Omega} \partial_{r}-\frac{6(g+2)}{r^{3}} H_{\Omega}\right\} \\
& -\sin 3 \phi M_{\Omega}\left\{\frac{3}{r} \partial_{r}^{2}-\frac{9}{r^{2}} \partial_{r}+\frac{8}{r^{3}}+\frac{2}{r^{3}} H_{\Omega}\right\}
\end{aligned}
$$

where the angular intertwiner $M_{\Omega}$ (to be given shortly) has been used.

Reducing to the angular subsystem, i.e. the Pöschl-Teller model, the formula (2.38) yields

$$
\mathcal{M}_{\Omega} \equiv \mathcal{M}_{1} \sim x^{1} \mathcal{D}_{2}-x^{2} \mathcal{D}_{1}+x^{2} \mathcal{D}_{3}-x^{3} \mathcal{D}_{2}+x^{3} \mathcal{D}_{1}-x^{1} \mathcal{D}_{3} \sim \mathrm{i}\left(w \mathcal{D}_{w}-\bar{w} \mathcal{D}_{\bar{w}}\right),
$$

which simplifies to

$$
\mathcal{M}_{1} \sim x^{1} \partial_{23}+x^{2} \partial_{31}+x^{3} \partial_{12}+g\left\{\frac{x^{1}+x^{2}-2 x^{3}}{x^{12}} s_{12}+\frac{x^{2}+x^{3}-2 x^{1}}{x^{23}} s_{23}+\frac{x^{3}+x^{1}-2 x^{2}}{x^{31}} s_{31}\right\}
$$

or to

$$
\mathcal{M}_{1} \sim \mathrm{i}\left(w \partial_{w}-\bar{w} \partial_{\bar{w}}\right)-\mathrm{i} g\left\{\frac{w-\bar{w}}{w+\bar{w}} s_{0}+\frac{\rho w-\bar{\rho} \bar{w}}{\rho w+\bar{\rho} \bar{w}} s_{+}+\frac{\bar{\rho} w-\rho \bar{w}}{\bar{\rho} w+\rho \bar{w}} s_{-}\right\} .
$$

Taking the residue, we arrive at

$$
M_{\Omega} \equiv M_{1} \sim x^{1} \partial_{23}+x^{2} \partial_{31}+x^{3} \partial_{12}-g \frac{\left(x^{1}+x^{2}-2 x^{3}\right)\left(x^{2}+x^{3}-2 x^{1}\right)\left(x^{3}+x^{1}-2 x^{2}\right)}{x^{12} x^{23} x^{31}}
$$

or, in complex and polar parametrizations,

$$
M_{1} \sim \mathrm{i}\left(w \partial_{w}-\bar{w} \partial_{\bar{w}}\right)-3 \mathrm{i} g \frac{w^{3}-\bar{w}^{3}}{w^{3}+\bar{w}^{3}}=\partial_{\phi}+3 g \tan 3 \phi=\cos ^{g}(3 \phi) \partial_{\phi} \cos ^{-g}(3 \phi) .
$$

\footnotetext{
${ }^{6}$ We remark that $\mathcal{D}_{w}^{3}+\mathcal{D}_{\bar{w}}^{3}$ changes sign under any Weyl reflection while $\mathcal{D}_{w}^{3}-\mathcal{D}_{\bar{w}}^{3}$ is totally symmetric.
} 
The penultimate expression is the familiar first-order operator which enjoys the following remarkable properties:

- it adds zeros at $\phi=0, \frac{2 \pi}{3}, \frac{4 \pi}{3}$ and poles at $\phi=\frac{\pi}{3}, \pi, \frac{5 \pi}{3}$

- it intertwines between symmetric and antisymmetric Weyl group singlets

- acting $\ell_{3}+1$ times on $v_{\ell}^{(0)}$ gives zero

- acting with $M_{1}^{(1)}=M_{1}^{(-1)^{\dagger}}$ on $v_{\ell}^{(0)}$ yields non-normalizable wave functions $v_{\ell}^{(-1)}$

- it is a Vandermonde dressing of the angular momentum

- it is the unique angular intertwiner

For $g=0$, the expression (3.9) is readily evaluated (with $r^{0} \rightarrow \ln r$ and $\ell=3 \ell_{3}$ ),

$$
v_{\ell}^{(0)} \sim r^{\ell}\left(\partial_{w}^{3}-\partial_{\bar{w}}^{3}\right)^{\ell_{3}} \ln w \bar{w} \sim r^{\ell}\left(w^{-\ell} \pm \bar{w}^{-\ell}\right) \sim\left\{\begin{array}{ll}
\cos \ell \phi & \text { for } \ell \text { even } \\
\sin \ell \phi & \text { for } \ell \text { odd }
\end{array},\right.
$$

which are all Weyl symmetric. Acting repeatedly with $M_{1}$ on these wave functions, one alternates between Weyl symmetric and Weyl antisymmetric and generates the wave functions at the same value of $\ell$ for any integer coupling. The low-lying states for $g=0,1,2,3$ are tabulated in table 1 . The action of the intertwiners is illustrated in figure 1 . The only exception is the one-dimensional kernel of $M_{1}$, consisting of the ground state

$$
v_{0}^{(g)} \sim r^{-3 g} \Delta^{g} \sim \cos ^{g} 3 \phi
$$

Finally, the additional conserved quantity $R_{2}$ manufactured with $M_{\Omega}$ and its adjoint, yields nothing new since

$$
R_{2}^{(g)}=\left(\partial_{\phi}-3 g \tan 3 \phi\right)\left(\partial_{\phi}+3 g \tan 3 \phi\right)=-2 H_{\Omega}^{(g)}+9 g^{2}=-\operatorname{res}\left(\mathcal{L}^{2}\right)=-C_{2}^{(g)},
$$

which provides $\rho_{2}^{(g+1)}=9$. We read off the intertwining relation

$$
M_{1}^{(g)} R_{2}^{(g)}=\left(R_{2}^{(g+1)}-9(1+2 g)\right) M_{1}^{(g)} .
$$

For generic values of $g$, there can be no further independent conserved quantity. However, when $g$ is integer, one additional algebraically independent conserved charge can be constructed,

$$
Q^{(g)}=M_{1}^{(g-1)} M_{1}^{(g-2)} \cdots M_{1}^{(1-g)} \quad \Longrightarrow \quad Q^{(g)} H_{\Omega}^{(g)}=Q^{(g)} H_{\Omega}^{(1-g)}=H_{\Omega}^{(g)} Q^{(g)},
$$

which is a Weyl-antiinvariant differential operator of order $2 g-1$ that squares to

$$
\left(Q^{(g)}\right)^{2}=\prod_{j=1-g}^{g-1}\left(-2 H_{\Omega}^{(g)}+9 j^{2}\right)
$$




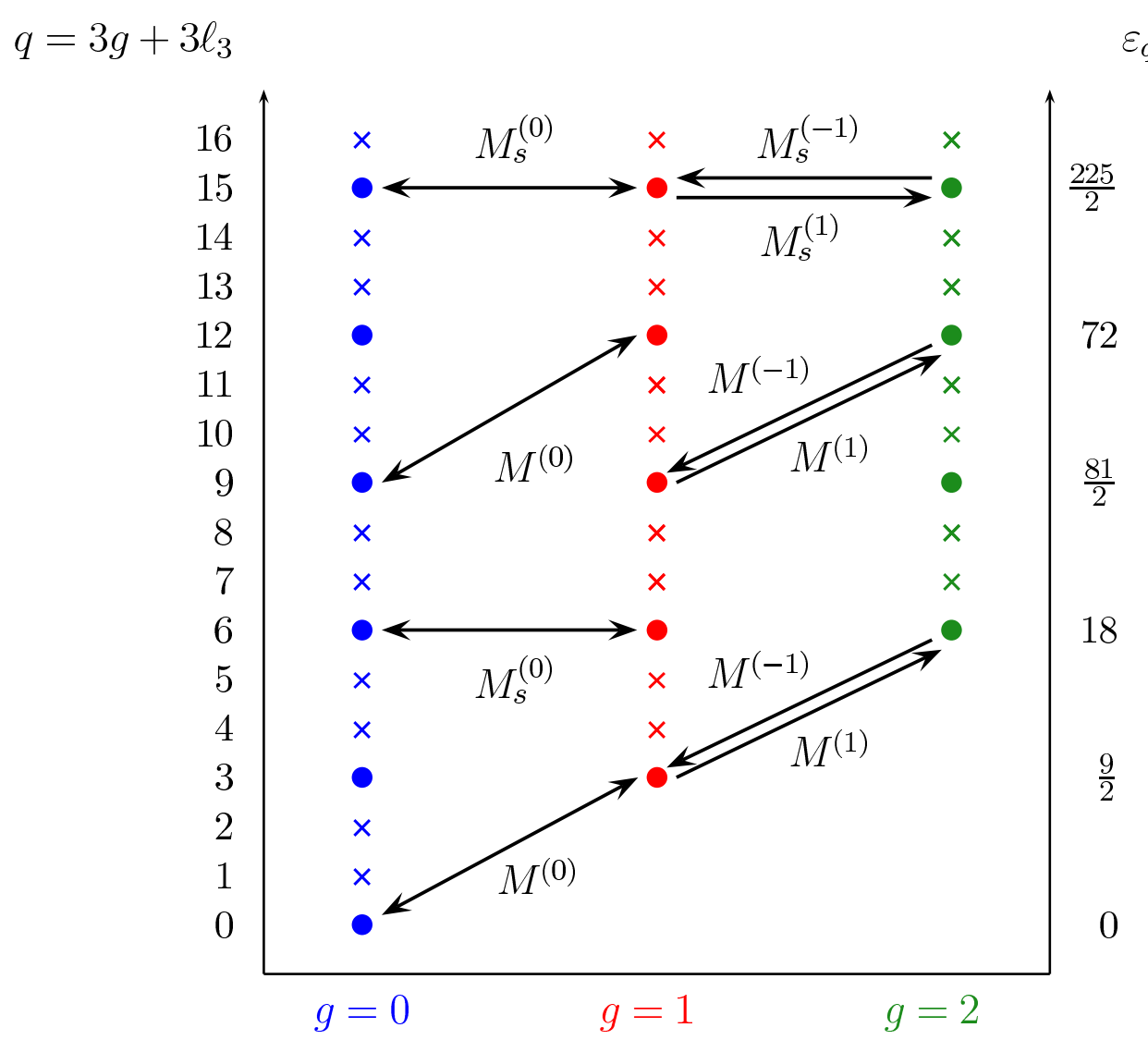

Figure 1. Pöschl-Teller model: spectrum and intertwiner actions.

\section{Tetrahexahedric model: the spectrum}

The main purpose of this paper is to work out the angular (relative) rational 4-particle (or $A_{3}$ ) Calogero model as the simplest non-separable case, in order to demonstrate the viability of the general observations of section 2 . We take advantage of the isometry $A_{3} \simeq D_{3}$ and employ $D_{3}$-type relative coordinates. ${ }^{7}$

To construct the model and find its spectrum, we introduce not Jacobi but WalshHadamard coordinates $\left\{y^{i}\right\}$ and their derivatives together with the center of mass $X$ via

$$
\begin{array}{lll}
x^{1}=X+\frac{1}{2}\left(+y^{1}+y^{2}+y^{3}\right), & \partial_{x^{1}}=\frac{1}{4} \partial_{X}+\frac{1}{2}\left(+\partial_{y^{1}}+\partial_{y^{2}}+\partial_{y^{3}}\right), \\
x^{2}=X+\frac{1}{2}\left(+y^{1}-y^{2}-y^{3}\right), & \partial_{x^{2}}=\frac{1}{4} \partial_{X}+\frac{1}{2}\left(+\partial_{y^{1}}-\partial_{y^{2}}-\partial_{y^{3}}\right), \\
x^{3}=X+\frac{1}{2}\left(-y^{1}+y^{2}-y^{3}\right), & \partial_{x^{3}}=\frac{1}{4} \partial_{X}+\frac{1}{2}\left(-\partial_{y^{1}}+\partial_{y^{2}}-\partial_{y^{3}}\right), \\
x^{4}=X+\frac{1}{2}\left(-y^{1}-y^{2}+y^{3}\right), & \partial_{x^{4}}=\frac{1}{4} \partial_{X}+\frac{1}{2}\left(-\partial_{y^{1}}-\partial_{y^{2}}+\partial_{y^{3}}\right) .
\end{array}
$$

The polar (or spherical) parametrization of $\mathbb{R}^{3}$ defines the radius $r$ and two angles $\theta$ and $\phi$,

$$
y^{1}=r \sin \theta \cos \phi, \quad y^{2}=r \sin \theta \sin \phi, \quad y^{3}=r \cos \theta .
$$

\footnotetext{
${ }^{7}$ See also [34] for an application of the same idea.
} 
To avoid index cluttering, we redenote the Euclidean $\mathbb{R}^{3}$ coordinates in a traditional fashion,

$$
\left(y^{1}, y^{2}, y^{3}\right)=:(x, y, z) .
$$

The angular momentum components read (up to a factor of i)

$$
L_{x}=-\left(y \partial_{z}-z \partial_{y}\right), \quad L_{y}=-\left(z \partial_{x}-x \partial_{z}\right), \quad L_{z}=-\left(x \partial_{y}-y \partial_{x}\right)
$$

so that (minus) the Laplacian on $S^{2}$ takes the form

$$
L^{2}=-\left(L_{x}^{2}+L_{y}^{2}+L_{z}^{2}\right)=-\frac{1}{\sin \theta} \partial_{\theta} \sin \theta \partial_{\theta}-\frac{1}{\sin ^{2} \theta} \partial_{\phi}^{2},
$$

and the (reduced) Hamiltonian becomes

$$
H=-\frac{1}{2}\left(\partial_{x}^{2}+\partial_{y}^{2}+\partial_{z}^{2}\right)+2 g(g-1)\left(\frac{x^{2}+y^{2}}{\left(x^{2}-y^{2}\right)^{2}}+\frac{y^{2}+z^{2}}{\left(y^{2}-z^{2}\right)^{2}}+\frac{z^{2}+x^{2}}{\left(z^{2}-x^{2}\right)^{2}}\right) .
$$

After the standard similarity transformation, we obtain

$$
H^{\prime} u \mapsto-\frac{1}{2} \partial_{r}^{2} u+\frac{1}{r^{2}} H_{\Omega} u \quad \text { and } \quad H_{\Omega}=\frac{1}{2} L^{2}+U
$$

with

$$
U(\theta, \phi)=2 g(g-1)\left\{\frac{1}{\sin ^{2} \theta \cos ^{2} 2 \phi}+\frac{\cos ^{2} \theta+\sin ^{2} \theta \cos ^{2} \phi}{\left(\cos ^{2} \theta-\sin ^{2} \theta \cos ^{2} \phi\right)^{2}}+\frac{\cos ^{2} \theta+\sin ^{2} \theta \sin ^{2} \phi}{\left(\cos ^{2} \theta-\sin ^{2} \theta \sin ^{2} \phi\right)^{2}}\right\} .
$$

This has been named 'cuboctahedric Higgs oscillator' potential [22] since it is the superposition of six Higgs oscillators placed at the positive roots of $A_{3} \simeq D_{3}$, and those (together with their antipodes) form the vertices of a cuboctahedron [35]. However, a better name is 'tetrahexahedral' potential, because the intersections of the Weyl chamber walls with the two-sphere form six great circles, which trace the 36 edges of a tetrahexahedron (or tetrakis hexahedron) [36]. Its 24 identical faces are isosceles triangles. The spherical projection tessellates the two-sphere into 24 spherically triangular domains in which the particle is trapped. This sequence of geometrical configurations, starting from the $A_{3}$ root system (cuboctahedron) and ending at the walls of the angular potential (tetrahexahedron) is displayed in figure 2, and color and contour plots of the potential are shown in figure 3 .

The angular energy eigenvalues become

$$
\varepsilon_{q}=\frac{1}{2} q(q+1) \quad \text { with } \quad q=6 g+\ell=6 g+3 \ell_{3}+4 \ell_{4} .
$$

The interaction reduces the $2 \ell+1$ dimensional free eigenspace (spanned by the spherical harmonics $Y_{\ell m}$ ) to the subspace invariant under the $S_{4}$ Weyl group action generated e.g. by

$$
\begin{array}{llll}
s_{x+y}:(x, y, z) & \mapsto(-y,-x,+z), & & s_{x-y}:(x, y, z) \mapsto(+y,+x,+z), \\
s_{y+z}:(x, y, z) \mapsto(+x,-z,-y), & & s_{y-z}:(x, y, z) \mapsto(+x,+z,+y), \\
s_{z+x}:(x, y, z) \mapsto(-z,+y,-x), & & s_{z-x}:(x, y, z) \mapsto(+z,+y,+x) .
\end{array}
$$

This reduces the degeneracy to $\operatorname{deg}\left(\varepsilon_{q}\right)=\operatorname{deg}_{4}(\ell)$ of (2.19). The corresponding wave functions

$$
\Psi_{E, q}(r, \theta, \phi)=j_{q}(\sqrt{2 E} r) v_{q}(\theta, \phi)
$$



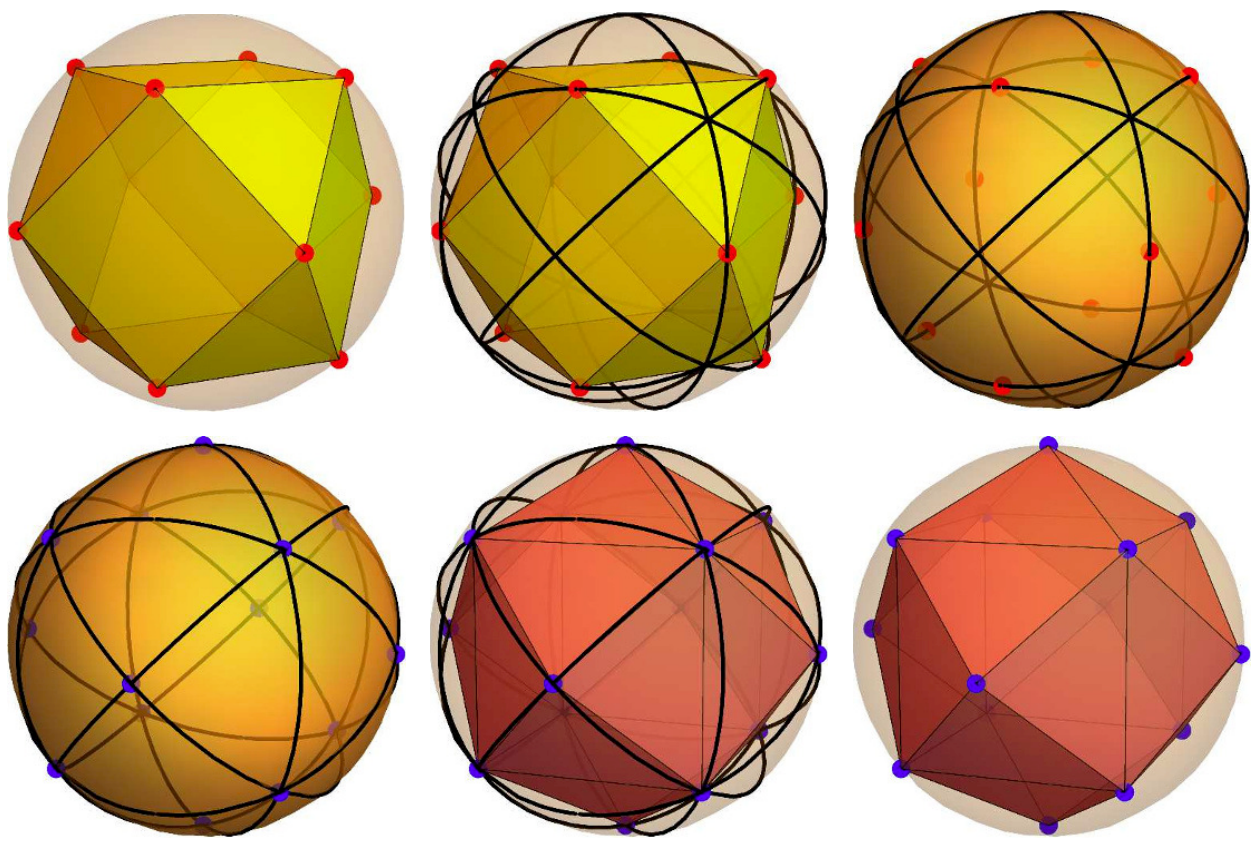

Figure 2. From the cuboctahedron to the tetrahexahedron.
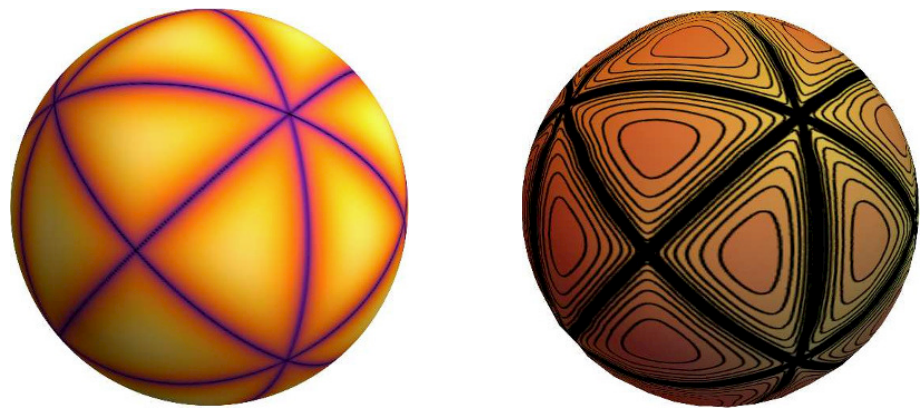

Figure 3. The tetrahexahedric potential (its $\log \log \log$ ) on the two-sphere.

have a spherical Bessel function as their radial part and involve two specific Weyl-symmetric homogeneous Dunkl polynomials, of degree three and four,

$$
\begin{aligned}
v_{q}(\theta, \phi) \equiv v_{\ell}^{(g)}(\theta, \phi) & \sim r^{q+1}\left(\sum_{\mu=1}^{3} \mathcal{D}_{\mu}^{3}\right)^{\ell_{3}}\left(\sum_{\nu=1}^{3} \mathcal{D}_{\nu}^{4}\right)^{\ell_{4}} \Delta^{g} r^{-1-12 g} \\
& \sim r^{q+1}\left(\mathcal{D}_{x} \mathcal{D}_{y} \mathcal{D}_{z}\right)^{\ell_{3}}\left(\mathcal{D}_{x}^{4}+\mathcal{D}_{y}^{4}+\mathcal{D}_{z}^{4}\right)^{\ell_{4}} \Delta^{g} r^{1-12 g},
\end{aligned}
$$

as well as the coordinate Vandermonde,

$$
\begin{aligned}
\Delta & =\prod_{i<j}\left(\left(y^{i}\right)^{2}-\left(y^{j}\right)^{2}\right)=\left(x^{2}-y^{2}\right)\left(x^{2}-z^{2}\right)\left(y^{2}-z^{2}\right) \\
& \sim r^{6} \sin ^{2} \theta \cos ^{4} \theta \cos ^{2} 2 \phi\left(\tan ^{2} \theta \cos ^{2} \phi-1\right)\left(\tan ^{2} \theta \sin ^{2} \phi-1\right) .
\end{aligned}
$$


Here, the three Dunkl operators on $\mathbb{R}^{3}$ are given by

$$
\begin{aligned}
& \mathcal{D}_{x}=\partial_{x}-\frac{g}{x+y} s_{x+y}-\frac{g}{x-y} s_{x-y}-\frac{g}{x+z} s_{x+z}-\frac{g}{x-z} s_{z-x}, \\
& \mathcal{D}_{y}=\partial_{y}-\frac{g}{y+x} s_{x+y}-\frac{g}{y-x} s_{x-y}-\frac{g}{y+z} s_{y+z}-\frac{g}{y-z} s_{y-z}, \\
& \mathcal{D}_{z}=\partial_{z}-\frac{g}{z+x} s_{z+x}-\frac{g}{z-x} s_{z-x}-\frac{g}{z+y} s_{y+z}-\frac{g}{z-y} s_{y-z} .
\end{aligned}
$$

The angular wave functions $v_{q}(\theta, \phi)$ in (4.12) are known as 'tetrahedral harmonics' in theoretical chemistry $[37,38] .{ }^{8}$ The low-lying states for $g=0,1,2$ are tabulated in table 2 .

For completeness, we here display some quantities in the potential-free frame. The potential-free Hamiltonian

$$
\widetilde{H}=-\frac{1}{2}\left(\partial_{x}^{2}+\partial_{y}^{2}+\partial_{z}^{2}\right)-\left(\frac{2 g x}{x^{2}-y^{2}}+\frac{2 g x}{x^{2}-z^{2}}\right) \partial_{x}-\left(\frac{2 g y}{y^{2}-z^{2}}+\frac{2 g y}{y^{2}-x^{2}}\right) \partial_{y}-\left(\frac{2 g z}{z^{2}-x^{2}}+\frac{2 g z}{z^{2}-y^{2}}\right) \partial_{z}
$$

annihilates the corresponding Dunkl-deformed harmonics

$$
\widetilde{h}_{\ell_{3}, \ell_{4}}^{(g)} \sim r^{12 g+1+6 \ell_{3}+8 \ell_{4}} \Delta^{-g}\left(\mathcal{D}_{x} \mathcal{D}_{y} \mathcal{D}_{z}\right)^{\ell_{3}}\left(\mathcal{D}_{x}^{4}+\mathcal{D}_{y}^{4}+\mathcal{D}_{z}^{4}\right)^{\ell_{4}} \Delta^{g} r^{1-12 g},
$$

so that the complete wave function may be written as

$$
\Psi_{E, \ell_{3}, \ell_{4}}^{(g)}(x, y, z)=j_{q}(\sqrt{2 E} r) r^{-q} \Delta^{g} \widetilde{h}_{\ell_{3}, \ell_{4}}^{(g)}(x, y, z) \quad \text { with } \quad q=6 g+3 \ell_{3}+4 \ell_{4} .
$$

\section{Tetrahexahedric model: intertwiners \& integrability}

According to (2.34), the four-particle intertwiner is

$$
M \sim \operatorname{res}\left(\left(\mathcal{D}_{x}^{2}-\mathcal{D}_{y}^{2}\right)\left(\mathcal{D}_{y}^{2}-\mathcal{D}_{z}^{2}\right)\left(\mathcal{D}_{z}^{2}-\mathcal{D}_{x}^{2}\right)\right)
$$

and it acts on the wave functions as

$$
M^{(g)} j_{q}(\sqrt{2 E} r) v_{\ell}^{(g)}(\theta, \phi) \sim j_{q+6}(\sqrt{2 E} r) v_{\ell}^{(g+1)}(\theta, \phi)
$$

The rather complicated explicit form of this six-order partial differential operator has been constructed in [19]. Let us turn our attention to the angular intertwiners $M_{s}$. Their building blocks are the Dunkl-deformed angular momenta

$$
\mathcal{L}_{x} \equiv \mathcal{L}_{y z}=-\left(y \mathcal{D}_{z}-z \mathcal{D}_{y}\right), \quad \mathcal{L}_{y} \equiv \mathcal{L}_{z x}=-\left(z \mathcal{D}_{x}-x \mathcal{D}_{z}\right), \quad \mathcal{L}_{z} \equiv \mathcal{L}_{x y}=-\left(x \mathcal{D}_{y}-y \mathcal{D}_{x}\right)
$$

or their complex combinations $\mathcal{L}_{ \pm}=\mathcal{L}_{x} \pm \mathrm{i} \mathcal{L}_{y}$. Employing (4.4) and (4.14), they read

$$
\begin{aligned}
& \mathcal{L}_{x}=L_{x}+g\left\{\frac{z}{x-y} s_{x-y}-\frac{z}{x+y} s_{x+y}-\frac{y+z}{y-z} s_{y-z}+\frac{y-z}{y+z} s_{y+z}+\frac{y}{z-x} s_{z-x}+\frac{y}{z+x} s_{z+x}\right\}, \\
& \mathcal{L}_{y}=L_{y}+g\left\{\frac{x}{y-z} s_{y-z}-\frac{x}{y+z} s_{y+z}-\frac{z+x}{z-x} s_{z-x}+\frac{z-x}{z+x} s_{z+x}+\frac{z}{x-y} s_{x-y}+\frac{z}{y+x} s_{x+y}\right\}, \\
& \mathcal{L}_{z}=L_{z}+g\left\{\frac{y}{z-x} s_{z-x}-\frac{y}{z+x} s_{z+x}-\frac{x+y}{x-y} s_{x-y}+\frac{x-y}{x+y} s_{x+y}+\frac{x}{y-z} s_{y-z}+\frac{x}{z+y} s_{y+z}\right\}
\end{aligned}
$$

\footnotetext{
${ }^{8}$ The chemistry literature seems to be unaware of the straightforward construction scheme (4.12).
} 
and transform under the Weyl reflections in the following fashion,

$$
\begin{aligned}
& s_{x+y}:\left(\mathcal{L}_{x}, \mathcal{L}_{y}, \mathcal{L}_{z}\right) \mapsto\left(+\mathcal{L}_{y},+\mathcal{L}_{x},-\mathcal{L}_{z}\right), \quad s_{x-y}:\left(\mathcal{L}_{x}, \mathcal{L}_{y}, \mathcal{L}_{z}\right) \mapsto\left(-\mathcal{L}_{y},-\mathcal{L}_{x},-\mathcal{L}_{z}\right), \\
& s_{y+z}:\left(\mathcal{L}_{x}, \mathcal{L}_{y}, \mathcal{L}_{z}\right) \mapsto\left(-\mathcal{L}_{x},+\mathcal{L}_{z},+\mathcal{L}_{y}\right), \quad s_{y-z}:\left(\mathcal{L}_{x}, \mathcal{L}_{y}, \mathcal{L}_{z}\right) \mapsto\left(-\mathcal{L}_{x},-\mathcal{L}_{z},-\mathcal{L}_{y}\right), \\
& s_{z+x}:\left(\mathcal{L}_{x}, \mathcal{L}_{y}, \mathcal{L}_{z}\right) \mapsto\left(+\mathcal{L}_{z},-\mathcal{L}_{y},+\mathcal{L}_{x}\right), \quad s_{z-x}:\left(\mathcal{L}_{x}, \mathcal{L}_{y}, \mathcal{L}_{z}\right) \mapsto\left(-\mathcal{L}_{z},-\mathcal{L}_{y},-\mathcal{L}_{x}\right)
\end{aligned}
$$

For convenience, we also note their transformation under certain even Weyl group elements,

$$
\begin{aligned}
& s_{x+y} s_{x-y}:\left(\mathcal{L}_{x}, \mathcal{L}_{y}, \mathcal{L}_{z}\right) \mapsto\left(-\mathcal{L}_{x},-\mathcal{L}_{y},+\mathcal{L}_{z}\right), \\
& s_{y+z} s_{y-z}:\left(\mathcal{L}_{x}, \mathcal{L}_{y}, \mathcal{L}_{z}\right) \mapsto\left(+\mathcal{L}_{x},-\mathcal{L}_{y},-\mathcal{L}_{z}\right), \\
& s_{z+x} s_{z-x}:\left(\mathcal{L}_{x}, \mathcal{L}_{y}, \mathcal{L}_{z}\right) \mapsto\left(-\mathcal{L}_{x},+\mathcal{L}_{y},-\mathcal{L}_{z}\right) .
\end{aligned}
$$

The construction (2.38) then yields a simple cubic polynomial in the angular Dunkl operators,

$$
\mathcal{M}_{\Omega} \equiv \mathcal{M}_{3}=\frac{1}{6}\left(\mathcal{L}_{x} \mathcal{L}_{y} \mathcal{L}_{z}+\mathcal{L}_{x} \mathcal{L}_{z} \mathcal{L}_{y}+\mathcal{L}_{y} \mathcal{L}_{z} \mathcal{L}_{x}+\mathcal{L}_{y} \mathcal{L}_{x} \mathcal{L}_{z}+\mathcal{L}_{z} \mathcal{L}_{x} \mathcal{L}_{y}+\mathcal{L}_{z} \mathcal{L}_{y} \mathcal{L}_{x}\right)
$$

which indeed is an anti-invariant under the Weyl group. It remains to evaluate the residue $M_{\Omega}=\operatorname{res}\left(\mathcal{M}_{\Omega}\right)$ for arriving at

$$
\begin{aligned}
M_{3}= & \frac{1}{6}\left(L_{x} L_{y} L_{z}+L_{x} L_{z} L_{y}\right)-2 g \frac{x y}{x^{2}-y^{2}}\left(L_{x} L_{y}+L_{y} L_{x}\right) \\
& +\left[16 g^{2} \frac{x^{2}}{\left(x^{2}-y^{2}\right)\left(z^{2}-x^{2}\right)}-4 g(g-1) \frac{x^{2}}{\left(y^{2}-z^{2}\right)^{2}}+2 g\left(\frac{1}{x^{2}-y^{2}}-\frac{1}{z^{2}-x^{2}}\right)\right] y z L_{x} \\
& +2 g(g-1)(g+2) x^{2}\left[\frac{y^{2}+z^{2}}{\left(y^{2}-z^{2}\right)^{2}}+z\left(\frac{1}{(y-z)^{3}}-\frac{1}{(y+z)^{3}}\right)\right]+g\left(2 g^{2}+8 g-1\right) \frac{y^{2}+z^{2}}{y^{2}-z^{2}} \\
& +2 g^{2}(8+9 g) \frac{x^{2} y^{2} z^{2}}{\left(x^{2}-y^{2}\right)\left(x^{2}-z^{2}\right)\left(y^{2}-z^{2}\right)}-\frac{2}{3} g^{3} \frac{x^{6}+y^{6}+z^{6}}{\left(x^{2}-y^{2}\right)\left(x^{2}-z^{2}\right)\left(y^{2}-z^{2}\right)}+\text { cyclic permutations . }
\end{aligned}
$$

Inserting the free angular momentum differential operators (4.4), we finally obtain

$$
\begin{aligned}
M_{3}= & y^{2} z \partial_{z x x}-y z^{2} \partial_{x x y}+\frac{1}{2}\left(y^{2}-z^{2}\right) \partial_{x x}+4 g \frac{y z}{y^{2}-z^{2}}\left(y z \partial_{x x}-z x \partial_{x y}+x^{2} \partial_{y z}-x y \partial_{z x}\right) \\
& +g\left[2 y^{2} z^{2}\left(\frac{8 g}{\left(x^{2}-y^{2}\right)\left(z^{2}-x^{2}\right)}+\frac{16 g}{\left(z^{2}-x^{2}\right)\left(y^{2}-z^{2}\right)}-\frac{2 g-1}{\left(x^{2}-y^{2}\right)^{2}}+\frac{2 g-1}{\left(z^{2}-x^{2}\right)^{2}}\right)\right. \\
& \left.-\frac{2 x^{2} y^{2}}{\left(z^{2}-x^{2}\right)^{2}}+\frac{2 x^{2} z^{2}}{\left(x^{2}-y^{2}\right)^{2}}-\frac{2 y^{2}}{x^{2}-y^{2}}-\frac{2 z^{2}}{z^{2}-x^{2}}-2 \frac{y^{2}+z^{2}}{y^{2}-z^{2}}\right] x \partial_{x} \\
& +2 g(g-1)(g+2) x^{2}\left[\frac{y^{2}+z^{2}}{\left(y^{2}-z^{2}\right)^{2}}+z\left(\frac{1}{(y-z)^{3}}-\frac{1}{(y+z)^{3}}\right)\right]+g\left(2 g^{2}+8 g-1\right) \frac{y^{2}+z^{2}}{y^{2}-z^{2}} \\
& +2 g^{2}(8+9 g) \frac{x^{2} y^{2} z^{2}}{\left(x^{2}-y^{2}\right)\left(x^{2}-z^{2}\right)\left(y^{2}-z^{2}\right)}-\frac{2}{3} g^{3} \frac{x^{6}+y^{6}+z^{6}}{\left(x^{2}-y^{2}\right)\left(x^{2}-z^{2}\right)\left(y^{2}-z^{2}\right)}+\text { cyclic permutations } .
\end{aligned}
$$

This is not the only elementary intertwiner however. At order six, one finds the next Weyl-antiinvariant function of $\left\{\mathcal{L}_{x}, \mathcal{L}_{y}, \mathcal{L}_{z}\right\}$, namely

$$
\mathcal{M}_{6}=\left\{\mathcal{L}_{x}^{4}, \mathcal{L}_{y}^{2}\right\}-\left\{\mathcal{L}_{y}^{4}, \mathcal{L}_{x}^{2}\right\}+\left\{\mathcal{L}_{y}^{4}, \mathcal{L}_{z}^{2}\right\}-\left\{\mathcal{L}_{z}^{4}, \mathcal{L}_{y}^{2}\right\}+\left\{\mathcal{L}_{z}^{4}, \mathcal{L}_{x}^{2}\right\}-\left\{\mathcal{L}_{x}^{4}, \mathcal{L}_{z}^{2}\right\}
$$

Its residue then produces a second intertwiner,

$$
\begin{aligned}
M_{6}= & \left\{L_{x}^{4}, L_{y}^{2}\right\}+4 g\left(\frac{x}{y+z}-\frac{x z}{z^{2}-x^{2}}\right)\left\{L_{x}^{4}, L_{y}\right\}+4 g\left(\frac{y z}{x^{2}-y^{2}}+\frac{y(y-2 z)}{y^{2}-z^{2}}\right)\left\{L_{x}^{3}, L_{y}^{2}\right\} \\
& +O\left(L_{x}^{4}, L_{x}^{3} L_{y}, L_{x}^{2} L_{y}^{2}\right) \pm \text { permutations } .
\end{aligned}
$$




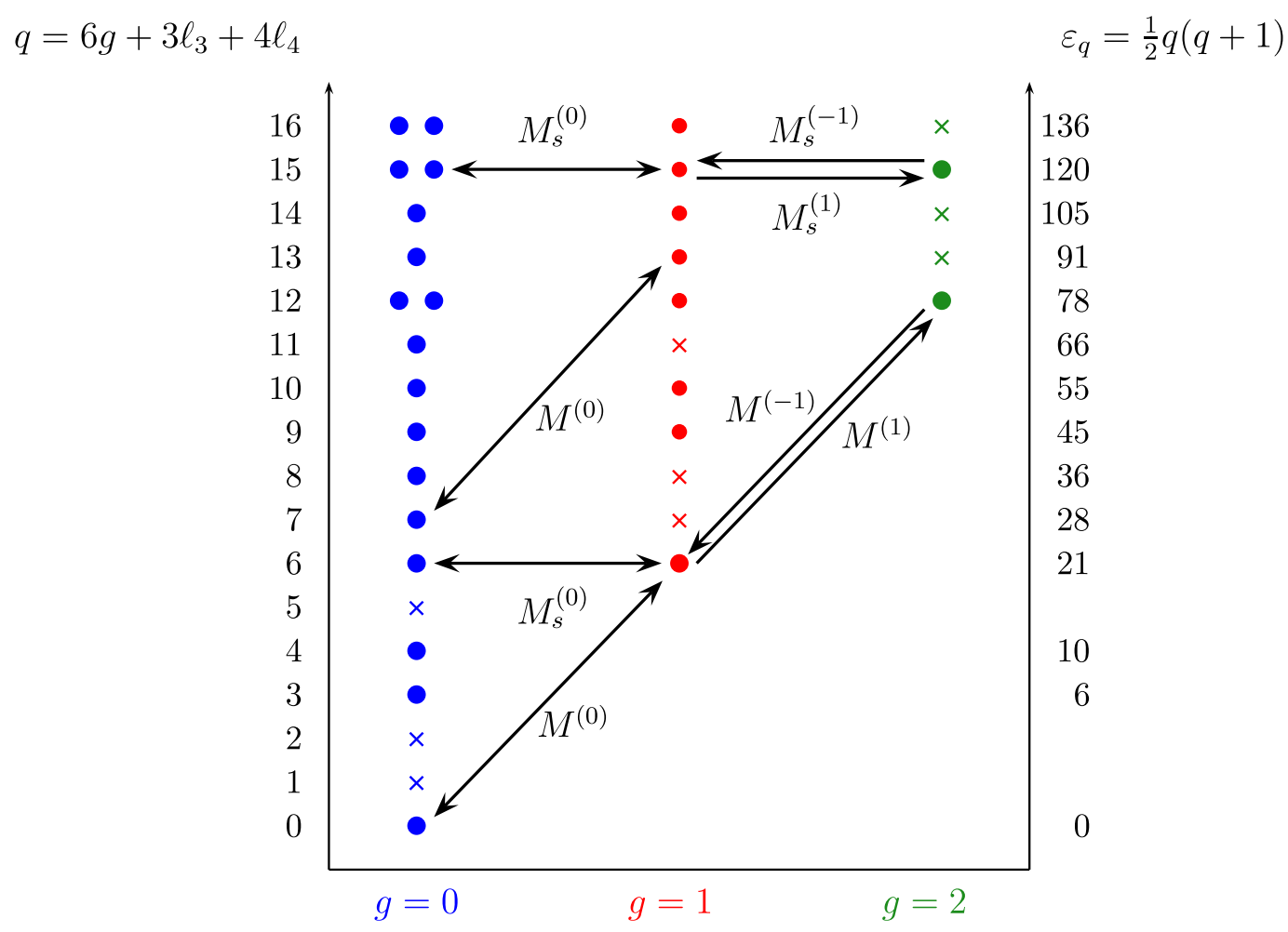

Figure 4. Tetrahexahedric model: spectrum and intertwiner actions.

The complete expression is too lengthy to display in this paper. We have checked that all higher-order intertwiners, generated from further Weyl-antiinvariants, can be decomposed into linear combinations of $M_{3}$ and $M_{6}$ with Weyl-symmetric functions of $\left\{\mathcal{L}_{x}, \mathcal{L}_{y}, \mathcal{L}_{z}\right\}$ as coefficients. In this sense, $M_{3}$ and $M_{6}$ algebraically generate the set of all intertwiners for $H_{\Omega}$. As a curiosity, we report a remarkable (non-local) example from this set,

$$
\begin{aligned}
\mathcal{M}_{\text {non-local }} & =\sin \mathcal{L}_{+} \sinh \mathcal{L}_{+}+\sin \mathcal{L}_{-} \sinh \mathcal{L}_{-}=\sum_{k=0}^{\infty}(-1)^{k} \frac{\mathcal{L}_{+}^{4 k+2}+\mathcal{L}_{-}^{4 k+2}}{(2 k+1) !(4 k+1) ! !} \\
& =\left(\mathcal{L}_{+}^{2}+\mathcal{L}_{-}^{2}\right)-\frac{1}{90}\left(\mathcal{L}_{+}^{6}+\mathcal{L}_{-}^{6}\right)+\frac{1}{113400}\left(\mathcal{L}_{+}^{10}+\mathcal{L}_{-}^{10}\right)-\frac{1}{681080400}\left(\mathcal{L}_{+}^{14}+\mathcal{L}_{-}^{14}\right)+\ldots
\end{aligned}
$$

In all cases, the action on the angular states is as expected,

$$
M_{s}^{(g)} v_{\ell}^{(g)}(\theta, \phi) \sim v_{\ell-6}^{(g+1)}(\theta, \phi),
$$

and the size of the kernels $\operatorname{dim} \operatorname{ker} M_{s}=\operatorname{dim} \operatorname{ker}_{4}(\ell)$ is independent of $g$ as was given in (2.43). The action of the intertwiners $M_{3}$ and $M_{6}$ as well as $M$ (5.2) is illustrated in figure 4 .

What about additional conserved charges in our tetrahexahedral angular Calogero system? The $S_{4}$ Weyl group action (5.5) on the Dunkl angular momenta is sufficiently simple to characterize the algebra of Weyl-symmetric polynomials. It is generated by

$$
J_{k}:=\operatorname{res}\left(\mathcal{L}_{x}^{k}+\mathcal{L}_{y}^{k}+\mathcal{L}_{z}^{k}\right) \quad \text { for } \quad k=0,2,4,6,
$$


and its center is formed by

$$
J_{0}=C_{0}=1 \quad \text { and } \quad J_{2}=-C_{2}=-2 H_{\Omega}+6 g(6 g+1),
$$

so $\left[J_{4}, H_{\Omega}\right]=\left[J_{6}, H_{\Omega}\right]=0$ but $\left[J_{6}, J_{4}\right] \neq 0$. Any word formed from $J_{4}$ and $J_{6}$ will commute with $H_{\Omega}$. The commutator $\left[J_{6}, J_{4}\right]$ cannot be expressed in terms of lower charges but is related to a Weyl-invariant product of the two intertwiners,

$$
\begin{aligned}
{\left[J_{6}, J_{4}\right]=} & 12 M_{3}^{\dagger} M_{6}+24(3+4 g) J_{6} J_{2}-12(3+2 g) J_{4} J_{4}-48(1+2 g) J_{4} J_{2} J_{2} \\
& +12(1+2 g) J_{2} J_{2} J_{2} J_{2}+24\left(97+113 g-96 g^{2}-144 g^{3}\right) J_{6} \\
& +12\left(266+349 g-304 g^{2}-528 g^{3}\right) J_{4} J_{2}+12\left(76+101 g-80 g^{2}-144 g^{3}\right) J_{2} J_{2} J_{2} \\
& -48\left(134+275 g-1015 g^{2}-1596 g^{3}+1008 g^{4}+1728 g^{5}\right) J_{4} \\
& +24\left(146+379 g-690 g^{2}-1240 g^{3}+672 g^{4}+1152 g^{5}\right) J_{2} J_{2} \\
& +96\left(10-39 g-775 g^{2}-2232 g^{3}-1584 g^{4}\right) J_{2} .
\end{aligned}
$$

Higher-degree invariants are not independent, e.g.

$$
\begin{aligned}
6 J_{8}= & 8 J_{6} J_{2}+3 J_{4} J_{4}-6 J_{4} J_{2} J_{2}+J_{2} J_{2} J_{2} J_{2} \\
& -12\left(8+5 g+12 g^{2}\right) J_{6}+4\left(34+23 g+30 g^{2}\right) J_{4} J_{2}-8\left(5+3 g+3 g^{2}\right) J_{2} J_{2} J_{2} \\
& +24\left(13+15 g-102 g^{2}-72 g^{3}\right) J_{4}-4\left(43+70 g-252 g^{2}-144 g^{3}\right) J_{2} J_{2} \\
& -48(1+3 g)(1+4 g)(1-12 g) J_{2} .
\end{aligned}
$$

It is instructive to work out the complete set of elementary intertwinings:

$$
\begin{aligned}
M_{3}^{(g)} J_{2}^{(g)}= & \left(J_{2}^{(g+1)}-6(7+12 g)\right) M_{3}^{(g)} \\
M_{3}^{(g)} J_{4}^{(g)}= & \left(J_{4}^{(g+1)}-4(11+12 g) J_{2}^{(g+1)}+48\left(26+73 g+48 g^{2}\right)\right) M_{3}^{(g)}+2 M_{6}^{(g)}, \\
M_{3}^{(g)} J_{6}^{(g)}= & \left(J_{6}^{(g+1)}-(35+36 g) J_{4}^{(g+1)}-3(7+4 g) J_{2}^{(g+1)} J_{2}^{(g+1)}\right. \\
& +2\left(1111+2668 g+1392 g^{2}\right) J_{2}^{(g+1)} \\
& \left.+96\left(457+1933 g+2717 g^{2}+1368 g^{3}+144 g^{4}\right)\right) M_{3}^{(g)} \\
& +\left(3 J_{2}^{(g+1)}-\left(115+200 g+48 g^{2}\right)\right) M_{6}^{(g)}, \\
M_{6}^{(g)} J_{2}^{(g)}= & \left(J_{2}^{(g+1)}-6(7+12 g)\right) M_{6}^{(g)}, \\
M_{6}^{(g)} J_{4}^{(g)}=( & -144 J_{6}^{(g+1)}+144 J_{4}^{(g+1)} J_{2}^{(g+1)}-32 J_{2}^{(g+1)} J_{2}^{(g+1)} J_{2}^{(g+1)} \\
- & 48\left(53+75 g+36 g^{2}\right) J_{4}^{(g+1)}+16\left(77+99 g+36 g^{2}\right) J_{2}^{(g+1)} J_{2}^{(g+1)} \\
- & 96\left(261+1151 g+1320 g^{2}+432 g^{3}\right) J_{2}^{(g+1)} \\
+ & \left.2304\left(159+1352 g+3672 g^{2}+4269 g^{3}+2232 g^{4}+432 g^{5}\right)\right) M_{3}^{(g)} \\
+ & \left(J_{4}^{(g+1)}-4(29+36 g) J_{2}^{(g+1)}+72\left(27+139 g+160 g^{2}+48 g^{3}\right)\right) M_{6}^{(g)},
\end{aligned}
$$

with $M_{6}^{(g)} J_{6}^{(g)}$ displayed in the appendix. They have the general structure predicted in $(2.48)$. 
Any maximally abelian subalgebra is generated by the center plus one arbitrary further element, hence only one Liouville charge besides the angular Hamiltonian can exist. There is no preferred choice among the words in $J_{4}$ and $J_{6}$, but distinguished combinations are

$$
\begin{aligned}
R_{6} \equiv M_{3}^{\dagger} M_{3}= & \frac{1}{3} J_{6}-\frac{1}{2} J_{4} J_{2}+\frac{1}{6} J_{2} J_{2} J_{2}-\frac{1}{6}\left(11+16 g-48 g^{2}\right) J_{4} \\
& +\frac{1}{12}\left(13+24 g-48 g^{2}\right) J_{2} J_{2}+\frac{1}{3}(1+3 g)(1+4 g)(1-12 g) J_{2}, \\
R_{12} \equiv M_{6}^{\dagger} M_{6}= & -12 J_{6} J_{6}+12\left\{J_{6}, J_{4}\right\} J_{2}-\frac{16}{3} J_{6} J_{2} J_{2} J_{2}+2 J_{4} J_{4} J_{4}-14 J_{4} J_{4} J_{2} J_{2} \\
& +6 J_{4} J_{2} J_{2} J_{2} J_{2}-\frac{2}{3} J_{2} J_{2} J_{2} J_{2} J_{2} J_{2}+\text { lower-order terms },
\end{aligned}
$$

where the full expression can be found in the appendix. The corresponding intertwining relations involve a single intertwiner only and read

$$
\begin{aligned}
M_{3}^{(g)} R_{6}^{(g)}= & \left(R_{6}^{(g+1)}-(1+2 g) \rho_{6}^{(g+1)}\right) M_{3}^{(g)} \quad \text { and } \\
M_{6}^{(g)} R_{12}^{(g)}= & \left(R_{12}^{(g+1)}-(1+2 g) \rho_{12}^{(g+1)}\right) M_{6}^{(g)} \quad \text { with } \\
\frac{1}{36} \rho_{6}^{(g+1)}= & -4 J_{4}^{(g+1)}+4 J_{2}^{(g+1)} J_{2}^{(g+1)}-2\left(69+128 g+48 g^{2}\right) J_{2}^{(g+1)}+3(5+4 g)(7+12 g)(11+12 g) \\
\frac{1}{4} \rho_{12}^{(g+1)}= & -54\left\{J_{6}^{(g+1)}, J_{4}^{(g+1)}\right\}-12 J_{6}^{(g+1)} J_{2}^{(g+1)} J_{2}^{(g+1)}+294 J_{4}^{(g+1)} J_{4}^{(g+1)} J_{2}^{(g+1)} \\
& -172 J_{4}^{(g+1)} J_{2}^{(g+1)} J_{2}^{(g+1)} J_{2}^{(g+1)}-62 J_{2}^{(g+1)} J_{2}^{(g+1)} J_{2}^{(g+1)} J_{2}^{(g+1)} J_{2}^{(g+1)}+O(g) .
\end{aligned}
$$

Similarly, the combinations $M_{3}^{\dagger} M_{6}$ and $M_{6}^{\dagger} M_{3}$ intertwine with a single intertwiner on either side of the relation (albeit not the same one).

Because the tetrahexahedral system is just two-dimensional, the existence of two conserved charges in addition to the Hamiltonian establishes its superintegrability. Beyond this, one can construct, for integral values of $g$, further conserved quantities,

$$
Q^{(g)}=M_{*}^{(g-1)} M_{*}^{(g-2)} \cdots M_{*}^{(1-g)} \Longrightarrow Q^{(g)} H_{\Omega}^{(g)}=Q^{(g)} H_{\Omega}^{(1-g)}=H_{\Omega}^{(g)} Q^{(g)},
$$

where $M_{*}$ stands for either $M_{3}$ or $M_{6}$, like it was achieved for the full Calogero model in [19]. These introduce a $\mathbb{Z}_{2}$ grading, with $Q$ being odd and all previous charges being even. Clearly, the $Q$ charges are algebraically independent of the even ones, but we expect that any two of them are related by some combination of even charges, and so they represent only a single additional algebraically independent conserved quantity. The anticommutator of any two $Q$ charges is some specific polynomial in $\left\{J_{6}, J_{4}, J_{2}\right\}$ whose order grows linearly with $g$. These and the commutators with the even charges are complicated but can be worked out in principle.

\section{Outlook}

The model we have considered in this paper is a very special one, and our treatment profited from the isometry $A_{3} \sim D_{3}$. It will be quite interesting to find out how the structure generalizes, firstly, to higher $n$ in $A_{n-1}$ and, secondly, to general Coxeter systems of rank $r$. Clearly, for the angular system three objects are crucial:

- the ring of Weyl-symmetric polynomials in $\left\{\mathcal{L}_{i j}\right\}$ and its independent generators

- maximal abelian subalgebras of the ring of Weyl-symmetric polynomials in $\left\{\mathcal{L}_{i j}\right\}$

- the elementary Weyl-antiinvariant polynomials in $\left\{\mathcal{L}_{i j}\right\}$ 
Assuming superintegrability, we conjecture that the number of ring generators equals $2 r-3$ and $r-1$ for the first and second item, respectively, but one has to go to $n=5$ (or $r=4$ ) to really test the second assertion. Especially useful will be the answer to the third item, as different intertwiners can be employed to partially lift the growing degeneracy of the energy levels.

Naturally, an extension to trigonometric, hyperbolic or even elliptic angular Calogero systems may be contemplated. Finally, it will be fascinating to introduce a $\mathcal{P} \mathcal{T}$ deformation into the angular system $[39,40]$, because it will remove the codimension-one singular loci of the potential, thereby connect the $n$ ! disjoint particle sectors and also give meaning to $g<0$ states, which are needed for the action of the odd $Q$ charges in the spectrum. In fact, it was observed that the action of such kind of charges on physical states gets regularized when $\mathcal{P T}$ deformations are considered, like in the case of the complex trigonometric PöschI-Teller potential (which corresponds to the 3-particle case considered here) [41].

\section{Acknowledgments}

This work was partially supported by the Alexander von Humboldt Foundation under grant CHL 1153844 STP, by the Deutsche Forschungsgemeinschaft under grant LE 838/12-1. The Centro de Estudios Científicos (CECs) is funded by the Chilean government through the Centers of Excellence Base Financing Program of Conicyt. This article is based upon work from COST Action MP1405 QSPACE, supported by COST (European Cooperation in Science and Technology).

\section{A Complementary formulae}

We collect the more lengthy formulae here. The most complex elementary intertwining relation reads

$$
\begin{aligned}
M_{6}^{(g)} J_{6}^{(g)}= & \left(J_{6}^{(g+1)}+\Gamma_{66}^{64} J_{4}^{(g+1)}+\Gamma_{66}^{622} J_{2}^{(g+1)} J_{2}^{(g+1)}+\Gamma_{66}^{62} J_{2}^{(g+1)}+\Gamma_{66}^{60}\right) M_{6}^{(g)} \\
& +\left(-144 J_{6}^{(g+1)} J_{2}^{(g+1)}-36 J_{4}^{(g+1)} J_{4}^{(g+1)}+168 J_{4}^{(g+1)} J_{2}^{(g+1)} J_{2}^{(g+1)}\right. \\
& -36 J_{2}^{(g+1)} J_{2}^{(g+1)} J_{2}^{(g+1)} J_{2}^{(g+1)}+\Gamma_{66}^{36} J_{6}^{(g+1)}+\Gamma_{66}^{342} J_{4}^{(g+1)} J_{2}^{(g+1)} \\
& +\Gamma_{66}^{3222} J_{2}^{(g+1)} J_{2}^{(g+1)} J_{2}^{(g+1)}+\Gamma_{66}^{34} J_{4}^{(g+1)}+\Gamma_{66}^{322} J_{2}^{(g+1)} J_{2}^{(g+1)}+\Gamma_{66}^{34} J_{4}^{(g+1)} \\
& \left.+\Gamma_{66}^{322} J_{2}^{(g+1)} J_{2}^{(g+1)}+\Gamma_{66}^{32} J_{2}^{(g+1)}+\Gamma_{66}^{30}\right) M_{3}^{(g)}
\end{aligned}
$$

with the abbreviations

$$
\begin{aligned}
\Gamma_{66}^{64} & =-(107+84 g), \\
\Gamma_{66}^{622} & =-3(23+36 g), \\
\Gamma_{66}^{62} & =2\left(4411+13438 g+12432 g^{2}+3168 g^{3}\right), \\
\Gamma_{66}^{60} & =-24\left(4826+33989 g+71466 g^{2}+64056 g^{3}+25056 g^{4}+3456 g^{5}\right), \\
\Gamma_{66}^{36} & =48\left(266+255 g+36 g^{2}\right),
\end{aligned}
$$




$$
\begin{aligned}
\Gamma_{66}^{342} & =-48\left(329+306 g+60 g^{2}\right), \\
\Gamma_{66}^{3222}= & 16\left(271+245 g+48 g^{2}\right), \\
\Gamma_{66}^{34}= & 48\left(4486+10765 g+8184 g^{2}+2160 g^{3}\right), \\
\Gamma_{66}^{322}= & -16\left(8071+22919 g+20280 g^{2}+5616 g^{3}\right), \\
\Gamma_{66}^{32}= & 96\left(23702+151661 g+328148 g^{2}+317796 g^{3}+139680 g^{4}+22464 g^{5}\right), \\
\Gamma_{66}^{30}= & -2304\left(13134+126277 g+440735 g^{2}+759693 g^{3}+715926 g^{4}+372744 g^{5}\right. \\
& \left.+99360 g^{6}+10368 g^{7}\right) .
\end{aligned}
$$

The full expression for the distinguished conserved charge $R_{12} \equiv M_{6}^{\dagger} M_{6}$ is

$$
\begin{aligned}
R_{12}= & -12 J_{6} J_{6}+12\left(J_{6} J_{4}+J_{6} J_{4}\right) J_{2}-\frac{16}{3} J_{6} J_{2} J_{2} J_{2}+2 J_{4} J_{4} J_{4}-14 J_{4} J_{4} J_{2} J_{2}+6 J_{4} J_{2} J_{2} J_{2} J_{2} \\
& -\frac{2}{3} J_{2} J_{2} J_{2} J_{2} J_{2} J_{2}+\gamma_{6}^{64}\left(J_{6} J_{4}+J_{6} J_{4}\right)+\gamma_{6}^{622} J_{6} J_{2} J_{2}+\gamma_{6}^{442} J_{4} J_{4} J_{2}+\gamma_{6}^{4222} J_{4} J_{2} J_{2} J_{2} \\
& +\gamma_{6}^{22222} J_{2} J_{2} J_{2} J_{2} J_{2}+\gamma_{6}^{62} J_{6} J_{2}+\gamma_{6}^{44} J_{4} J_{4}+\gamma_{6}^{422} J_{4} J_{2} J_{2}+\gamma_{6}^{2222} J_{2} J_{2} J_{2} J_{2}+\gamma_{6}^{6} J_{6} \\
& +\gamma_{6}^{42} J_{4} J_{2}+\gamma_{6}^{222} J_{2} J_{2} J_{2}+\gamma_{6}^{4} J_{4}+\gamma_{6}^{22} J_{2} J_{2}+\gamma_{6}^{2} J_{2},
\end{aligned}
$$

where the $g$-dependent coefficients are

$$
\begin{aligned}
\gamma_{6}^{64} & =-4\left(140+39 g+36 g^{2}\right), \\
\gamma_{6}^{622} & =\frac{1120}{3}-24 g+96 g^{2}, \\
\gamma_{6}^{442} & =8\left(202+63 g+30 g^{2}\right), \\
\gamma_{6}^{4222} & =-\frac{8}{3}\left(388+65 g+48 g^{2}\right), \\
\gamma_{6}^{22222} & =\frac{16}{3}\left(31+3 g+3 g^{2}\right), \\
\gamma_{6}^{62} & =-64\left(322+203 g-210 g^{2}-216 g^{3}\right), \\
\gamma_{6}^{44} & =16\left(553+308 g-1884 g^{2}-648 g^{3}\right), \\
\gamma_{6}^{422} & =\frac{8}{3}\left(7045+4352 g+2712 g^{2}-4320 g^{3}\right), \\
\gamma_{6}^{2222} & =-\frac{8}{3}\left(2157+944 g+48 g^{2}-1296 g^{3}\right), \\
\gamma_{6}^{6} & =-96\left(3940+1469 g-8691 g^{2}-4584 g^{3}+5328 g^{4}+3456 g^{5}\right), \\
\gamma_{6}^{42} & =16\left(35466+18288 g-97805 g^{2}-54456 g^{3}+56880 g^{4}+44928 g^{5}\right), \\
\gamma_{6}^{222} & =-\frac{16}{3}\left(33074+18470 g-94827 g^{2}-41064 g^{3}+51984 g^{4}+38016 g^{5}\right), \\
\gamma_{6}^{4} & =192\left(5864+6158 g-57481 g^{2}-32799 g^{3}+119796 g^{4}+68112 g^{5}-60480 g^{6}-41472 g^{7}\right), \\
\gamma_{6}^{22} & =-32\left(19568+28410 g-165011 g^{2}-106098 g^{3}+323232 g^{4}+146592 g^{5}-138240 g^{6}-82944 g^{7}\right), \\
\gamma_{6}^{2} & =-384\left(448-2274 g-32767 g^{2}-58215 g^{3}+52068 g^{4}+64296 g^{5}-89856 g^{6}-51840 g^{7}\right) .
\end{aligned}
$$

Finally, the shift $\rho_{12}^{(g+1)}=\frac{1}{1+2 g}\left(R_{12}^{(g+1)}-R_{12}^{(-g)}\right)$ takes the form

$$
\begin{aligned}
\frac{1}{4} \rho_{12}^{(g+1)}= & -54\left(J_{6}^{(g+1)} J_{4}^{(g+1)}+J_{4}^{(g+1)} J_{6}^{(g+1)}\right)-12 J_{6}^{(g+1)} J_{2}^{(g+1)} J_{2}^{(g+1)}+294 J_{4}^{(g+1)} J_{4}^{(g+1)} J_{2}^{(g+1)} \\
& -172 J_{4}^{(g+1)} J_{2}^{(g+1)} J_{2}^{(g+1)} J_{2}^{(g+1)}-62 J_{2}^{(g+1)} J_{2}^{(g+1)} J_{2}^{(g+1)} J_{2}^{(g+1)} J_{2}^{(g+1)}+\eta_{12}^{62} J_{6}^{(g+1)} J_{2}^{(g+1)} \\
& +\eta_{12}^{44} J_{4}^{(g+1)} J_{4}^{(g+1)}+\eta_{12}^{422} J_{4}^{(g+1)} J_{2}^{(g+1)} J_{2}^{(g+1)}+\eta_{12}^{2222} J_{2}^{(g+1)} J_{2}^{(g+1)} J_{2}^{(g+1)} J_{2}^{(g+1)} \\
& +\eta_{12}^{6} J_{6}^{(g+1)}+\eta_{12}^{42} J_{4}^{(g+1)} J_{2}^{(g+1)}+\eta_{12}^{222} J_{2}^{(g+1)} J_{2}^{(g+1)} J_{2}^{(g+1)}+\eta_{12}^{4} J_{4}^{(g+1)} \\
& +\eta_{12}^{22} J_{2}^{(g+1)} J_{2}^{(g+1)}+\eta_{12}^{2} J_{2}^{(g+1)}+\eta_{12}^{0},
\end{aligned}
$$


and the nontrivial coefficients are as follows,

$$
\begin{aligned}
\eta_{12}^{62}= & 36\left(25+304 g+144 g^{2}\right), \\
\eta_{12}^{44}= & -3\left(1739+4008 g+1152 g^{2}\right), \\
\eta_{12}^{422}= & 2\left(737-3924 g-3168 g^{2}\right), \\
\eta_{12}^{2222}= & 10013+23048 g+2208 g^{2}, \\
\eta_{12}^{6}= & -24\left(274+8397 g+27252 g^{2}+22032 g^{3}+5184 g^{4}\right), \\
\eta_{12}^{42}= & 12\left(10096+71365 g+159372 g^{2}+118224 g^{3}+22464 g^{4}\right), \\
\eta_{12}^{222}= & -4\left(151692+632591 g+717108 g^{2}+116208 g^{3}+19008 g^{4}\right), \\
\eta_{12}^{4}= & -24\left(82623+628336 g+1923894 g^{2}+2950776 g^{3}+2270592 g^{4}+767232 g^{5}+124416 g^{6}\right), \\
\eta_{12}^{22}= & 12\left(1509081+9095222 g+18576756 g^{2}+13405872 g^{3}+1368576 g^{4}+442368 g^{5}+82944 g^{6}\right), \\
\eta_{12}^{2}= & -96\left(2838989+22731865 g+67596615 g^{2}+87293784 g^{3}+37690416 g^{4}-6715008 g^{5}-1824768 g^{6}\right), \\
\eta_{12}^{0}= & 1728\left(972216+9826815 g+39041714 g^{2}+75013828 g^{3}+65364036 g^{4}+10127664 g^{5}\right. \\
& \left.-15564096 g^{6}-4084992 g^{7}\right) .
\end{aligned}
$$




\begin{tabular}{|c|cccc|}
\hline$\ell$ & $v_{\ell}^{(0)}$ & $v_{\ell}^{(1)}$ & $v_{\ell}^{(2)}$ & $v_{\ell}^{(3)}$ \\
\hline 0 & 1 & $\mathrm{c}$ & $\mathrm{c}^{2}$ & $\mathrm{c}^{3}$ \\
3 & $\mathrm{~s}$ & $\mathrm{c} \mathrm{s}$ & $\mathrm{c}^{2} \mathrm{~s}$ & $\mathrm{c}^{3} \mathrm{~s}$ \\
6 & $\mathrm{c}^{2}-\mathrm{s}^{2}$ & $\mathrm{c}\left(\mathrm{c}^{2}-3 \mathrm{~s}^{2}\right)$ & $\mathrm{c}^{2}\left(\mathrm{c}^{2}-5 \mathrm{~s}^{2}\right)$ & $\mathrm{c}^{3}\left(\mathrm{c}^{2}-7 \mathrm{~s}^{2}\right)$ \\
9 & $3 \mathrm{c}^{2} \mathrm{~s}-\mathrm{s}^{3}$ & $\mathrm{c}\left(\mathrm{c}^{2} \mathrm{~s}-\mathrm{s}^{3}\right)$ & $\mathrm{c}^{2}\left(3 \mathrm{c}^{2} \mathrm{~s}-5 \mathrm{~s}^{3}\right)$ & $\mathrm{c}^{3}\left(3 \mathrm{c}^{2} \mathrm{~s}-7 \mathrm{~s}^{3}\right)$ \\
12 & $\mathrm{c}^{4}-6 \mathrm{c}^{2} \mathrm{~s}^{2}+\mathrm{s}^{4}$ & $\mathrm{c}\left(\mathrm{c}^{4}-10 \mathrm{c}^{2} \mathrm{~s}^{2}+5 \mathrm{~s}^{4}\right)$ & $\mathrm{c}^{2}\left(3 \mathrm{c}^{4}-42 \mathrm{c}^{2} \mathrm{~s}^{2}+35 \mathrm{~s}^{4}\right)$ & $\mathrm{c}^{3}\left(\mathrm{c}^{4}-18 \mathrm{c}^{2} \mathrm{~s}^{2}+21 \mathrm{~s}^{4}\right)$ \\
15 & $5 \mathrm{c}^{4} \mathrm{~s}-10 \mathrm{c}^{2} \mathrm{~s}^{3}+\mathrm{s}^{5}$ & $\mathrm{c}\left(3 \mathrm{c}^{4} \mathrm{~s}-10 \mathrm{c}^{2} \mathrm{~s}^{3}+3 \mathrm{~s}^{5}\right)$ & $\mathrm{c}^{2}\left(3 \mathrm{c}^{4} \mathrm{~s}-14 \mathrm{c}^{2} \mathrm{~s}^{3}+7 \mathrm{~s}^{5}\right)$ & $\mathrm{c}^{3}\left(5 \mathrm{c}^{4} \mathrm{~s}-30 \mathrm{c}^{2} \mathrm{~s}^{3}+21 \mathrm{~s}^{5}\right)$ \\
18 & $\mathrm{c}^{6}-15 \mathrm{c}^{4} \mathrm{~s}^{2}+15 \mathrm{c}^{2} \mathrm{~s}^{4}-\mathrm{s}^{6}$ & $\mathrm{c}\left(\mathrm{c}^{6}-21 \mathrm{c}^{4} \mathrm{~s}^{2}+35 \mathrm{c}^{2} \mathrm{~s}^{4}-7 \mathrm{~s}^{6}\right)$ & $\mathrm{c}^{2}\left(\mathrm{c}^{6}-27 \mathrm{c}^{4} \mathrm{~s}^{2}+63 \mathrm{c}^{2} \mathrm{~s}^{4}-21 \mathrm{~s}^{6}\right)$ & $\mathrm{c}^{3}\left(5 \mathrm{c}^{6}-165 \mathrm{c}^{4} \mathrm{~s}^{2}+495 \mathrm{c}^{2} \mathrm{~s}^{4}-231 \mathrm{~s}^{6}\right)$ \\
21 & $7 \mathrm{c}^{6} \mathrm{~s}-35 \mathrm{c}^{4} \mathrm{~s}^{3}+21 \mathrm{c}^{2} \mathrm{~s}^{5}-\mathrm{s}^{7}$ & $\mathrm{c}\left(\mathrm{c}^{6} \mathrm{~s}-7 \mathrm{c}^{4} \mathrm{~s}^{3}+7 \mathrm{c}^{2} \mathrm{~s}^{5}-\mathrm{s}^{7}\right)$ & $\mathrm{c}^{2}\left(5 \mathrm{c}^{6} \mathrm{~s}-45 \mathrm{c}^{4} \mathrm{~s}^{3}+63 \mathrm{c}^{2} \mathrm{~s}^{5}-15 \mathrm{~s}^{7}\right)$ & $\mathrm{c}^{3}\left(5 \mathrm{c}^{6} \mathrm{~s}-55 \mathrm{c}^{4} \mathrm{~s}^{3}+99 \mathrm{c}^{2} \mathrm{~s}^{5}-33 \mathrm{~s}^{7}\right)$ \\
\hline
\end{tabular}

Table 1. Low-lying wave functions $v_{\ell}^{(g)}$ of the Pöschl-Teller model. Notation: $\mathrm{s} \equiv \sin (3 \phi)$ and $\mathrm{c} \equiv \cos (3 \phi)$. 


\begin{tabular}{|c|cc|c|}
\hline$\ell$ & $\ell_{3}$ & $\ell_{4}$ & $\widetilde{h}_{\ell_{3}, \ell_{4}}^{(0)}$ \\
\hline 0 & 0 & 0 & $\{000\}$ \\
3 & 1 & 0 & $\{111\}$ \\
4 & 0 & 1 & $\{400\}-3\{220\}$ \\
6 & 2 & 0 & $\{600\}-15\{420\}+30\{222\}$ \\
7 & 1 & 1 & $3\{511\}-5\{331\}$ \\
8 & 0 & 2 & $\{800\}-28\{620\}+35\{440\}$ \\
9 & 3 & 0 & $9\{711\}-63\{531\}+70\{333\}$ \\
10 & 2 & 1 & $5\{911\}-60\{731\}+63\{522\}-630\{442\}$ \\
11 & 1 & 2 & $\{1000\}-45\{820\}+42\{640\}+504\{62\}$ \\
12 & 4 & 0 & $36\{1200\}-2376\{1020\}+2445\{840\}+46125\{822\}+4893\{660\}-215250\{642\}+179375\{444\}$ \\
12 & 0 & 3 & $101\{1200\}-6666\{1020\}+47100\{840\}+8685\{822\}-42609\{660\}-40530\{642\}+33775\{444\}$ \\
\hline
\end{tabular}

\begin{tabular}{|c|cc|c|}
\hline$\ell$ & $\ell_{3}$ & $\ell_{4}$ & $\widetilde{h}_{\ell_{3}, \ell_{4}}^{(1)}$ \\
\hline 0 & 0 & 0 & $\{000\}$ \\
3 & 1 & 0 & $\{111\}$ \\
4 & 0 & 1 & $3\{400\}-11\{220\}$ \\
6 & 2 & 0 & $3\{600\}-39\{420\}+196\{222\}$ \\
7 & 1 & 1 & $5\{511\}-13\{331\}$ \\
8 & 0 & 2 & $\{800\}-20\{620\}+23\{440\}+12\{422\}$ \\
9 & 3 & 0 & $3\{711\}-27\{531\}+56\{333\}$ \\
10 & 2 & 1 & $35\{911\}-476\{731\}+477\{551\}+204\{533\}$ \\
11 & 1 & 2 & $12\{1200\}-456\{1020\}+657\{840\}+13581\{822\}+1137\{660\}-88842\{642\}+114007\{444\}$ \\
12 & 4 & 0 & $813\{1200\}-30894\{1020\}+165652\{840\}+72131\{822\}-147943\{660\}-169702\{642\}+57527\{444\}$ \\
12 & 0 & 3 & \\
\hline
\end{tabular}

\begin{tabular}{|c|cc|c|}
\hline$\ell$ & $\ell_{3}$ & $\ell_{4}$ & $\widetilde{h}_{\ell_{3}, \ell_{4}}^{(2)}$ \\
\hline 0 & 0 & 0 & $\{000\}$ \\
3 & 1 & 0 & $\{111\}$ \\
4 & 0 & 1 & $5\{400\}-19\{220\}$ \\
6 & 2 & 0 & $5\{600\}-63\{420\}+506\{222\}$ \\
7 & 1 & 1 & $\{511\}-3\{331\}$ \\
8 & 0 & 2 & $35\{800\}-644\{620\}+729\{440\}+552\{422\}$ \\
9 & 3 & 0 & $21\{711\}-207\{531\}+634\{333\}$ \\
10 & 2 & 1 & $21\{911\}-300\{731\}+303\{551\}+200\{533\}$ \\
11 & 1 & 2 & $980\{1200\}-31752\{1020\}+51045\{840\}+1248957\{822\}+84757\{660\}-9568482\{642\}+16884639\{444\}$ \\
12 & 4 & 0 & $41000\}-25\{820\}+38\{640\}+592\{622\}-1374442\}$ \\
12 & 0 & 3 & $411005\{1200\}-13316562\{1020\}+65753340\{840\}+36003897\{822\}-58071653\{660\}-80991402\{642\}+7379439\{444\}$ \\
\hline
\end{tabular}

Table 2. Some wave functions $v_{\ell}^{(g)}=r^{-\ell-6 g} \Delta^{g} \widetilde{h}_{\ell}^{(g)}$ of the terahexahedric model. $\{r s t\}:=x^{r} y^{s} z^{t}+x^{r} y^{t} z^{s}+x^{s} y^{t} z^{r}+x^{s} y^{r} z^{t}+x^{t} y^{r} z^{s}+x^{t} y^{s} z^{r}$. 
Open Access. This article is distributed under the terms of the Creative Commons Attribution License (CC-BY 4.0), which permits any use, distribution and reproduction in any medium, provided the original author(s) and source are credited.

\section{References}

[1] F. Calogero, Solution of a three-body problem in one-dimension, J. Math. Phys. 10 (1969) 2191 [InSPIRE].

[2] F. Calogero, Solution of the one-dimensional $N$ body problems with quadratic and/or inversely quadratic pair potentials, J. Math. Phys. 12 (1971) 419 [INSPIRE].

[3] F. Calogero and C. Marchioro, Exact solution of a one-dimensional three-body scattering problem with two-body and/or three-body inverse-square potentials,

J. Math. Phys. 15 (1974) 1425 [INSPIRE].

[4] M.A. Olshanetsky and A.M. Perelomov, Classical integrable finite dimensional systems related to Lie algebras, Phys. Rept. 71 (1981) 313 [INSPIRE].

[5] M.A. Olshanetsky and A.M. Perelomov, Quantum integrable systems related to Lie algebras, Phys. Rept. 94 (1983) 313 [INSPIRE].

[6] A.P. Polychronakos, Exchange operator formalism for integrable systems of particles, Phys. Rev. Lett. 69 (1992) 703 [hep-th/9202057] [INSPIRE].

[7] A.P. Polychronakos, Physics and mathematics of Calogero particles, J. Phys. A 39 (2006) 12793 [hep-th/0607033] [InSPIRE].

[8] S. Wojciechowski, Superintegrability of the Calogero-Moser system, Phys. Lett. A 95 (1983) 279.

[9] V.B. Kuznetsov, Hidden symmetry of the quantum Calogero-Moser system, Phys. Lett. A 218 (1996) 212 [solv-int/9509001] [INSPIRE].

[10] G. Barucchi and T. Regge, Conformal properties of a class of exactly solvable N-body problems in space dimension one, J. Math. Phys. 18 (1977) 1149 [INSPIRE].

[11] O.A. Chalykh and A.P. Veselov, Commutative rings of partial differential operators and Lie algebras, Commun. Math. Phys. 126 (1990) 597.

[12] O.A. Chalykh, Additional integrals of the generalized quantum Calogero-Moser system, Theor. Math. Phys. 109 (1996) 1269.

[13] E.M. Opdam, Root systems and hypergeometric functions. III, Composito Math. 67 (1988) 21.

[14] E.M. Opdam, Root systems and hypergeometric functions. IV, Composito Math. 67 (1988) 191.

[15] G.J. Heckman, A remark on the Dunkl differential-difference operators, in Progerss in Mathematics. Vol. 101: Harmonic analysis on reductive groups, W. Barker and P. Sally eds., Birkhäuser, Boston U.S.A. (1991).

[16] C.F. Dunkl, Differential-difference operators associated to reflection groups, Trans. Amer. Math. Soc. 311 (1989) 167.

[17] C.F. Dunkl and Y. Xu, Orthogonal polynomials of several variables, Cambridge University Press, Cambridge U.K. (2001).

[18] N. Reshetikhin, Degenerately integrable systems, arXiv:1509.00730 [INSPIRE]. 
[19] F. Correa, O. Lechtenfeld and M. Plyushchay, Nonlinear supersymmetry in the quantum Calogero model, JHEP 04 (2014) 151 [arXiv:1312.5749] [INSPIRE].

[20] P.W. Higgs, Dynamical Symmetries in a Spherical Geometry. 1, J. Phys. A 12 (1979) 309 [INSPIRE].

[21] H.I. Leemon, Dynamical Symmetries in a Spherical Geometry. 2, J. Phys. A 12 (1979) 489 [INSPIRE].

[22] T. Hakobyan, A. Nersessian and V. Yeghikyan, Cuboctahedric Higgs oscillator from the Calogero model, J. Phys. A 42 (2009) 205206 [arXiv:0808.0430] [InSPIRE].

[23] T. Hakobyan, S. Krivonos, O. Lechtenfeld and A. Nersessian, Hidden symmetries of integrable conformal mechanical systems, Phys. Lett. A 374 (2010) 801 [arXiv:0908.3290] [INSPIRE].

[24] O. Lechtenfeld, A. Nersessian and V. Yeghikyan, Action-angle variables for dihedral systems on the circle, Phys. Lett. A 374 (2010) 4647 [arXiv: 1005.0464] [InSPIRE].

[25] T. Hakobyan, O. Lechtenfeld, A. Nersessian and A. Saghatelian, Invariants of the spherical sector in conformal mechanics, J. Phys. A 44 (2011) 055205 [arXiv: 1008.2912] [InSPIRE].

[26] T. Hakobyan, O. Lechtenfeld and A. Nersessian, The spherical sector of the Calogero model as a reduced matrix model, Nucl. Phys. B 858 (2012) 250 [arXiv:1110.5352] [INSPIRE].

[27] T. Hakobyan, O. Lechtenfeld, A. Nersessian, A. Saghatelian and V. Yeghikyan, Action-angle variables and novel superintegrable systems, Phys. Part. Nucl. 43 (2012) 577 [INSPIRE].

[28] M.V. Feigin, Intertwining relations for the spherical parts of generalized Calogero operators, Theor. Math. Phys. 135 (2003) 497.

[29] M. Feigin, O. Lechtenfeld and A.P. Polychronakos, The quantum angular Calogero-Moser model, JHEP 07 (2013) 162 [arXiv:1305.5841] [INSPIRE].

[30] M. Feigin and T. Hakobyan, On the algebra of Dunkl angular momentum operators, arXiv: 1409.2480 [INSPIRE].

[31] M.S. Plyushchay, Deformed Heisenberg algebra, fractional spin fields and supersymmetry without fermions, Annals Phys. 245 (1996) 339 [hep-th/9601116] [INSPIRE].

[32] M.S. Plyushchay, Hidden nonlinear supersymmetries in pure parabosonic systems, Int. J. Mod. Phys. A 15 (2000) 3679 [hep-th/9903130] [inSPIRE].

[33] H. Gupta, C.E. Gwyther and J.C.P. Miller, Royal Society Mathematical tables. Volume 4: Tables of Partitions, Cambridge University Press, Cambridge U.K. (1958).

[34] C.F. Dunkl, Some orthogonal polynomials in four variables, SIGMA 4 (2008) 82 [arXiv:0812.0063].

[35] Cuboctahedron, Wikipedia article, http://en.wikipedia.org/wiki/Cuboctahedron.

[36] Tetrakis hexahedron, Wikipedia article, http://en.wikipedia.org/wiki/Tetrakis_hexahedron.

[37] K. Foz and I. Ozier, Construction of tetrahedral harmonics, J. Chem. Phys. 52 (1970) 5044.

[38] P.E.S. Wormer, Tetrahedral harmonics revisited, Molec. Phys. 99 (2001) 1973.

[39] A. Fring, A note on the integrability of non-Hermitian extensions of Calogero-Moser-Sutherland models, Mod. Phys. Lett. A 21 (2006) 691 [hep-th/0511097] [INSPIRE].

[40] A. Fring, $\mathcal{P} \mathcal{T}$-symmetric deformations of integrable models, Phil. Trans. Roy. Soc. Lond. A 371 (2013) 20120046 [arXiv:1204.2291] [InSPIRE].

[41] F. Correa and M.S. Plyushchay, Spectral singularities in PT-symmetric periodic finite-gap systems, Phys. Rev. D 86 (2012) 085028 [arXiv:1208.4448] [InSPIRE]. 